\title{
NPM1-Mutated Myeloid Neoplasms with $<20 \%$ Blasts: A Really Distinct Clinico-Pathologic Entity?
}

\author{
Fabio Forghieri ${ }^{1}$, , Vincenzo Nasillo ${ }^{1}$, Ambra Paolini ${ }^{1}$, Francesca Bettelli ${ }^{1}$, Valeria Pioli ${ }^{1}$, \\ Davide Giusti ${ }^{1}$, Andrea Gilioli ${ }^{1}$, Corrado Colasante ${ }^{1}$, Gloria Acquaviva ${ }^{1,2}$, Giovanni Riva ${ }^{3}$, \\ Patrizia Barozzi ${ }^{1}{ }^{\infty}$, Rossana Maffei ${ }^{1} \mathbb{D}$, Leonardo Potenza ${ }^{1}$, Roberto Marasca ${ }^{1}(\mathbb{D}$, \\ Claudio Fozza ${ }^{2}$, Enrico Tagliafico ${ }^{4}$, Tommaso Trenti ${ }^{3}$, Patrizia Comoli ${ }^{5}$, Giuseppe Longo ${ }^{6}$ and \\ Mario Luppi ${ }^{1}$
}

1 Section of Hematology, Department of Medical and Surgical Sciences, University of Modena and Reggio Emilia, Azienda Ospedaliero-Universitaria di Modena, Policlinico, 41124 Modena, Italy; vincenzo.nasillo@unimore.it (V.N.); paolini.ambra@aou.mo.it (A.P.); francesca.bettelli@unimore.it (F.B.); pioli.valeria@aou.mo.it (V.P.); davide.giusti@unimore.it (D.G.); andrea.gilioli@unimore.it (A.G.); colasantecorrado@gmail.com (C.C.); gloriaacquaviva@gmail.com (G.A.); patrizia.barozzi@unimore.it (P.B.); rossana.maffei@unimore.it (R.M.); leonardo.potenza@unimore.it (L.P.); roberto.marasca@unimore.it (R.M.); mario.luppi@unimore.it (M.L.)

2 Department of Clinical and Experimental Medicine, University of Sassari, 07100 Sassari, Italy; cfozza@uniss.it

3 Department of Laboratory Medicine and Pathology, Unità Sanitaria Locale, 41126 Modena, Italy; g.riva@ausl.mo.it (G.R.); t.trenti@ausl.mo.it (T.T.)

4 Center for Genome Research, Department of Medical and Surgical Sciences, University of Modena and Reggio Emilia, Azienda Ospedaliero-Universitaria di Modena, 41124 Modena, Italy; enrico.tagliafico@unimore.it

5 Pediatric Hematology/Oncology Unit and Cell Factory, Istituto di Ricovero e Cura a Carattere Scientifico (IRCCS) Policlinico San Matteo, 27100 Pavia, Italy; pcomoli@smatteo.pv.it

6 Division of Oncologic Medicine, Department of Oncology and Hematology,

Azienda Ospedaliero-Universitaria di Modena, Policlinico, 41124 Modena, Italy; longo@unimore.it

* Correspondence: fabio.forghieri@unimore.it; Tel.: +39-059-422-2447; Fax: +39-059-422-2386

Received: 28 October 2020; Accepted: 24 November 2020; Published: 26 November 2020

\begin{abstract}
Nucleophosmin (NPM1) gene mutations rarely occur in non-acute myeloid neoplasms (MNs) with $<20 \%$ blasts. Among nearly 10,000 patients investigated so far, molecular analyses documented NPM1 mutations in around $2 \%$ of myelodysplastic syndrome (MDS) cases, mainly belonging to MDS with excess of blasts, and 3\% of myelodysplastic/myeloproliferative neoplasm (MDS/MPN) cases, prevalently classified as chronic myelomonocytic leukemia. These uncommon malignancies are associated with an aggressive clinical course, relatively rapid progression to overt acute myeloid leukemia (AML) and poor survival outcomes, raising controversies on their classification as distinct clinico-pathologic entities. Furthermore, fit patients with NPM1-mutated MNs with $<20 \%$ blasts could benefit most from upfront intensive chemotherapy for AML rather than from moderate intensity MDS-directed therapies, although no firm conclusion can currently be drawn on best therapeutic approaches, due to the limited available data, obtained from small and mainly retrospective series. Caution is also suggested in definitely diagnosing NPM1-mutated MNs with blast count $<20 \%$, since NPM1-mutated AML cases frequently present dysplastic features and multilineage bone marrow cells showing abnormal cytoplasmic NPM1 protein delocalization by immunohistochemical staining, therefore belonging to NPM1-mutated clone regardless of blast morphology. Further prospective studies are warranted to definitely assess whether NPM1 mutations may become sufficient to diagnose AML, irrespective of blast percentage.
\end{abstract}


Keywords: NPM1 mutation; myelodysplastic syndromes; myelodysplastic/myeloproliferative neoplasms; chronic myelomonocytic leukemia; acute myeloid leukemia; leukemogenesis

\section{Introduction}

The nucleophosmin (NPM1) gene encodes an ubiquitous protein which physiologically shuttles between the nucleus and cytoplasm, acting as a molecular chaperone to establish multiple protein-protein interactions [1]. The shuttling between different cell compartments of wild-type NPM1 protein is fairly regulated by specific signal motifs [2,3]. In details, NPM1 protein mainly localizes to the nucleolus through a nucleolar localization signal (NoLS) at the C-terminus containing two tryptophans, namely, W288 and W290, while the nuclear export of wild-type NPM1 is mediated by the interaction, with low affinity, between two N-terminal nuclear export signals (NES) and the nuclear exporter XPO1 [2-4]. The import and anchoring signals eventually exceed the export signals, so that at steady state, wild-type NPM1 protein shows predominant nucleolar localization [1-5]. NPM1 protein is normally involved in multiple critical cell functions, such as control of ribosome biogenesis and export, regulation of centrosome duplication and formation of the mitotic spindle, histone chaperoning, DNA repair through binding to TP53 and APE1 and therefore influencing their activities depending on the type of DNA damage [1-5]. More recently, a functional role of wild-type NPM1 protein has been recognized in facilitating the "liquid-liquid" phase separation process in the multilayered structure of the nucleolus [5-7]. Moreover, NPM1 protein participates in 2'-O-methylation of ribosomal RNA [5,8]. In more details, NPM1 directly binds to several C/D box small nucleolar RNAs (snoRNAs) and to the methyltransferase fibrillarin, forming a protein complex which actively methylates rRNA and regulates translation $[5,8]$.

The NPM1 gene translocations to different partner genes are implicated in the pathogenesis of several hematopoietic malignancies, including CD30-positive anaplastic large-cell lymphoma with $\mathrm{t}(2 ; 5)$, the infrequent myelodysplasia/acute myeloid leukemia (MDS/AML) with $\mathrm{t}(3 ; 5)$ and extremely rare cases of acute promyelocytic leukemia with $t(5 ; 17)$, resulting in the generation of NPM1-ALK, NPM1-MLF1 and NPM1-RARA fusion transcripts, respectively [2,3]. All these genetic alterations usually perturb the normal cellular traffic of NPM1 protein in malignant cells, but we will focus here on the biological and clinical significance of NPM1 gene mutations occurring in acute myeloid leukemia (AML) and other myeloid neoplasms [2-5].

\section{NPM1-Mutated Acute Myeloid Leukemia: Biological and Clinical Features}

NPM1 gene mutations, occurring in approximately $30 \%$ of adult AML cases, and in $50-60 \%$ of AML cases with normal karyotype, represent some of the most frequent molecular lesions documented in AML [5,9-11]. Since the discovery of heterozygous NPM1 mutations in 2005 by Falini et al. [12], more than 55 different mutations, mainly occurring in exon 12 of the gene, have been described, but three mutation types (A recognized as a duplication of TCTG tetranucleotide sequence at positions 956-959, B and D) account for nearly 95\% of all cases [4,5,13]. NPM1 gene mutations, probably arising from replication errors primed by an illegitimate terminal deoxynucleotidyl transferase (TdT) activity [14], result in structural changes of the C-terminus of NPM1 protein, with subsequent aberrant cytoplasmic delocalization, leading to a unique immunohistochemical pattern (NPMc+) detectable on BM trephine biopsy [5,12,15]. In details, loss of C-terminal W288 and/or W290 impairs the NPM1 protein ability to reside in the nucleolus, while concurrent generation of a new C-terminal NES with high affinity to XPO1, reinforces the export action of the N-terminal NESs, thus increasing the probability of NPM1 mutants to be exported to the cytoplasm [3,5,13]. Moreover, NPM1-mutated proteins dominantly act over wild-type NPM1, causing the formation of heterodimers with abnormal cytoplasmic delocalization in AML cells, so that NPM1-mutated proteins are generally considered as "born to be exported" [3,5,15]. Through binding to NPM1 mutants, also several nuclear proteins, 
including tumor suppressors and transcription factors, involved in regulation of apoptosis, DNA repair and differentiation, are aberrantly exported and delocalized to the cytoplasm, therefore causing perturbations of these multiple cellular pathways through a combination of loss of functions and gain of functions, critical for leukemogenesis [2-5,13]. Of note, it was recently reported that NPM1-mutated protein also co-dislocates PU.1 into cytoplasm, whereas CEBPA and RUNX1, the master transcription factors that collaborate with PU.1 to activate granulo-monocytic lineages, remain in the nucleus. However, without nuclear PU.1, their coregulator interactions are toggled from coactivators to corepressors, thus repressing $>500$ granulocyte and monocyte terminal differentiation genes [16]. Furthermore, NPM1-mutated AML shows up-regulation of HOX genes, mainly HOXA and HOXB, with an expression signature that is nearly identical to that observed in normal hematopoietic stem cells, suggesting a significant involvement in self-renewal capacity $[5,17,18]$. The histone modifier MLL1 contributes to regulating HOX genes expression in NPM1-mutated AML through the interaction between MLL1 and the co-factor Menin. It has recently been demonstrated that nuclear re-localization or targeted degradation of NPM1-mutated protein in vitro induces loss of HOX gene expression, leading to leukemic cell differentiation and growth arrest $[17,18]$. Therefore, even if precise molecular mechanisms still need to be elucidated, NPM1-mutated protein shows a gain of function activity upstream of HOX genes, contributing to maintain the undifferentiated state of leukemic cells $[5,17,18]$.

NPM1 gene mutations are stable over time, usually documented at disease relapse and commonly expressed in the whole leukemic population $[3-5,19,20]$. Of note, NPM1-mutated AML, usually de novo, showing unique genetic, pathologic, immunophenotypic and clinical features, has now been recognized as a full distinct entity among AML with recurrent genetic abnormalities in the 2016 revision of WHO classification of myeloid neoplasms (MNs) and acute leukemia [21]. While the presence of coexisting chromosomal abnormalities, observed in only $15 \%$ of patients, does not generally appear to modify the prognostic effects of NPM1 mutations $[10,22,23]$ except in rare cases when adverse-risk cytogenetic alterations are present with subsequent unfavorable prognosis [24], clinical outcomes may be significantly influenced by accompanying molecular lesions, mainly involving $F L T 3, I D H 1, I D H 2$ and DNMT3A genes $[5,9,10,13]$. In details, the better risk outcomes observed in NPM1-mutated AML adult patients are generally considered limited to cases without concurrent FLT3-ITD mutations [10,13,25]. Furthermore, the deleterious prognostic effects of FLT3-ITD have previously been found to be most clinically relevant when co-occurring with NPM1 and DNMT3A mutations, as opposed with either mutation alone [11]. Recent studies suggested that patients with NPM1 mutation and FLT3-ITD with a low $(<0.5)$ allelic ratio have a similar favorable outcome as patients with NPM1-mutated AML without FLT3-ITD [26-29]. Thus, both these latter subgroups are currently considered favorable according to the 2017 ELN risk stratification, contrary to NPM1-mutated AML associated with FLT3-ITD with high allelic ratio, which is characterized by a higher relapse rate and poorer overall survival (OS) [10]. However, further studies did not confirm the favorable outcome of NPM1-mutated AML patients with FLT3-ITD low allelic ratio, at least when treated with intensive chemotherapy alone [30-32], while the addition of midostaurin significantly improved outcomes also in this latter genotype [33]. Thus, the risk-stratification according to FLT3-ITD allelic ratio still remains controversial and needs to be further validated, especially investigating the clinical role of FLT3 tyrosine-kinase inhibitors and allogeneic hematopoietic stem cell transplantation (HSCT) in different genetic subgroups [5,34]. Of note, several clinical trials have so far demonstrated a significant independent prognostic impact of minimal/measurable residual disease (MRD) monitoring in NPM1-mutated AML by highly-sensitive real-time quantitative polymerase chain reaction (RQ-PCR) and, accordingly, NPM1-mutated AML patients should have quantitative MRD assessment at informative clinical timepoints during treatment and follow-up to optimize anti-leukemic therapeutic strategies [20,35]. However, several controversies still remain, mainly regarding the most clinically significant timepoints and MRD thresholds to be considered, but also relating to the optimal source to be analyzed, namely, bone marrow (BM) or peripheral blood (PB) samples, and the correlation of MRD with other known prognostic indicators [20]. 


\section{Frequency of NPM1 Mutations in Patients with Non-Acute Myeloid Neoplasms}

As mentioned above, in the seminal study by Falini et al., NPMc+ was documented by immunohistochemical examination in 208 of 591 (35.2\%) BM trephine biopsy specimens obtained from patients with de novo AML, whereas normal nuclear NPM1 protein localization was found in 135 secondary AML (sAML) specimens and in 980 hematopoietic or extra-hematopoietic neoplasms other than AML, including acute lymphoblastic leukemia, chronic myeloid leukemia, non-Hodgkin lymphoma and myelodysplastic syndrome (MDS) cases [12]. Furthermore, the finding that NPM1 was neither mutated nor ectopically expressed in the cytoplasm in any human neoplasm different from AML was subsequently confirmed by immunohistochemical and/or mutational analyses carried out on more than 5000 samples [3,36]. However, it should be noted that, though less frequently compared to de novo AML, NPM1 mutations have also been observed in SAML, progressing from either MDS or myelodysplastic/myeloproliferative neoplasms (MDS/MPN), with a variable incidence around $10-15 \%$ and ranging from $4.5 \%$ to $27 \%$ of the cases [26-44]. Of note, Schnittger et al. detected NPM1 mutations in 67 of $504(13.3 \%)$ patients with sAML [38,39] and backtracked the mutation by RQ-PCR on paired samples collected at both initial diagnosis and AML evolution in 21 cases. Interestingly, NPM1 mutations were already detectable during the MDS phase in 15 cases, namely at very low levels $(0.01-1 \%)$ in 8 patients and at a median level $10 \%$ (range 5-100\%), comparable to that observed in sAML phase, in 7 patients. Conversely, in 6 patients, NPM1 mutations were not found during the antecedent MDS phase [38,39], as also reported by Courville et al. [40], therefore suggesting, before the identification of dynamics of hematopoietic clones characterized by next generation sequencing (NGS) assays, that this molecular lesion could also be acquired during the transformation process as a secondary event, at least in rare cases of sAML arising from MDS or MDS/MPN [38-40].

Furthermore, since the discovery of NPM1 mutations in AML, several studies have molecularly investigated the presence of NPM1 mutations in non-acute MNs with $<20 \%$ blasts, namely, MDS and MDS/MPN cases, either by single-gene PCR assays or by newer high-throughput molecular techniques, such as NGS, as summarized in Table $1[37,40,44-86]$. Among nearly 10,000 patients with MNs with $<20 \%$ blasts so far evaluated, the prevalence of NPM1 mutations was generally low, ranging from $0 \%$ to $11 \%$, with an overall frequency of $2 \%$ (Table 1 ) [37,40,44-86]. In more details, NPM1 mutations have collectively been reported by molecular analyses in nearly $2 \%$ of patients diagnosed with MDS, mainly belonging to high-risk cases, categorized as MDS with excess of blasts (MDS-EB), and in around 3\% of MDS/MPN cases, mainly classified as chronic myelomonocytic leukemia (CMML), as summarized in Tables 1 and 2 [37,40,44-86]. 
Table 1. Prevalence of NPM1 mutations in patients with MDS or MDS/MPN: review of the literature.

\begin{tabular}{|c|c|c|c|c|c|c|c|c|c|}
\hline Reference & Type of Study & $\begin{array}{l}\text { Number of } \\
\text { Cases }\end{array}$ & $\begin{array}{l}\text { Median Age } \\
\text { (Range), } \\
\text { Years }\end{array}$ & $\operatorname{Sex}(M / F)$ & $\begin{array}{l}\text { Molecular } \\
\text { Analysis }\end{array}$ & $\begin{array}{l}\text { Overall Frequency of } \\
\text { NPM1 Mutations } \\
\text { Analyzed in MNs } \\
\text { with }<20 \% \text { Blasts }\end{array}$ & $\begin{array}{l}\text { Frequency of NPM1 } \\
\text { Mutations in } \\
\text { MDS Cases }\end{array}$ & $\begin{array}{l}\text { Frequency of NPM1 } \\
\text { Mutations in } \\
\text { MDS/MPN Cases }\end{array}$ & $\begin{array}{l}\text { Frequency of NPM1 } \\
\text { Mutations in } \\
\text { CMML Cases }\end{array}$ \\
\hline Caudill et al., 2006 [45] & Monocenter & 90 & NA & NA & PCR & $3 / 90(3.3 \%)$ & $0 / 30$ & $3 / 60(5 \%)$ & $3 / 60(5 \%)$ \\
\hline Oki et al., 2006 [46] & $\begin{array}{l}\text { Monocenter, } \\
\text { retrospective }\end{array}$ & $199 *$ & NA & NA & PCR & $2 / 115(1.7 \%)$ & $0 / 50$ & $2 / 65(3.1 \%)$ & $2 / 50(4 \%)$ \\
\hline Zhang et al., 2007 [47] & Monocenter & 38 & NA & NA & PCR & $2 / 38(5.2 \%)$ & $2 / 38(5.2 \%)$ & - & - \\
\hline Shiseki et al., 2007 [48] & Monocenter & $28^{\wedge}$ & $70(29-85) \S$ & $17 / 11 \S$ & PCR & $0 / 16$ & $0 / 16$ & - & - \\
\hline Ishikawa et al., 2008 [49] & Monocenter & 36 & $58(28-89)$ & $24 / 12$ & PCR & $2 / 36(5.5 \%)$ & $2 / 36(5.5 \%)$ & - & - \\
\hline Andersen et al., 2008 [50] & $\begin{array}{l}\text { Monocenter, } \\
\text { retrospective }\end{array}$ & $140^{\wedge}$ & 61 & $67 / 73$ & PCR & $3 / 89(3.4 \%)^{\circ}$ & $3 / 89(3.4 \%)^{\circ}$ & - & - \\
\hline Bacher et al., 2009 [51] & $\begin{array}{l}\text { Monocenter, } \\
\text { retrospective }\end{array}$ & 166 & NA & NA & PCR & $2 / 166(1.2 \%)$ & $2 / 149(1.3 \%)$ & $0 / 17$ & $0 / 17$ \\
\hline Chen et al., 2009 [52] & $\begin{array}{l}\text { Monocenter, } \\
\text { retrospective }\end{array}$ & 29 & $62(22-77)$ & $18 / 11$ & PCR & $0 / 29$ & - & $0 / 29$ & $0 / 29$ \\
\hline Ernst et al., 2010 [53] & $\begin{array}{l}\text { Multicenter, } \\
\text { retrospective }\end{array}$ & 187 & NA & NA & PCR & $6 / 187(3.2 \%)$ & - & $6 / 187(3.2 \%)$ & $6 / 97(6.2 \%)$ \\
\hline Rocquain et al., 2010 [54] & $\begin{array}{l}\text { Multicenter, } \\
\text { retrospective }\end{array}$ & $129^{\wedge}$ & NA & NA & NGS/PCR & $0 / 65$ & $0 / 65$ & - & - \\
\hline Li et al., 2010 [55] & - & 232 & NA & NA & PCR & 9/232 (3.9\%) & 9/232 (3.9\%) & - & - \\
\hline Thol et al., 2010 [56] & $\begin{array}{l}\text { Multicenter, } \\
\text { retrospective }\end{array}$ & 193 & $65(36-92)$ & $119 / 74$ & PCR & $1 / 193(0.5 \%)$ & $1 / 193(0.5 \%)$ & - & - \\
\hline Gritsaev et al., 2010 [57] & - & 44 & NA & NA & PCR & $5 / 44(11.4 \%)$ & - & - & - \\
\hline Gelsi-Boyer et al., 2010 [58] & $\begin{array}{l}\text { Multicenter, } \\
\text { prospective }\end{array}$ & 53 & $71(41-88)$ & $36 / 17$ & PCR & $0 / 49$ & - & $0 / 49$ & $0 / 49$ \\
\hline Dicker et al., 2010 [37] & Monocenter & $269^{\wedge}$ & 69.4 & $120 / 82$ & PCR & 1/73 (1.4\%) & $1 / 66(1.5 \%)$ & $0 / 7$ & $0 / 7$ \\
\hline Bejar et al., 2011 [59] & $\begin{array}{l}\text { Multicenter, } \\
\text { retrospective }\end{array}$ & 439 & 70 & $306 / 133$ & NGS & $8 / 439(1.8 \%)$ & $8 / 439(1.8 \%)$ & - & - \\
\hline Bacher et al., 2011 [60] & $\begin{array}{l}\text { Monocenter, } \\
\text { retrospective }\end{array}$ & $212^{\wedge}$ & $68.8(18-88) \S$ & $139 / 73 \S$ & PCR & $3 / 34(8.8 \%)$ & $3 / 34(8.8 \%)$ & - & - \\
\hline Machado-Neto et al., 2011 [61] & Monocenter & $51^{\wedge}$ & $63(26-90) \S$ & $30 / 21 \S$ & PCR & $0 / 46$ & $0 / 46$ & - & - \\
\hline Bains et al., 2011 [62] & $\begin{array}{l}\text { Monocenter, } \\
\text { retrospective }\end{array}$ & 160 & $68(22-89)$ & $98 / 62$ & PCR & $7 / 160(4.4 \%)$ & $4 / 139(2.9 \%)$ & $3 / 21(14.3 \%)$ & $2 / 15(13.3 \%)$ \\
\hline Papaemmanuil et al., 2013 [63] & Multicenter & $738^{\wedge}$ & $\begin{array}{c}68 \pm 13 \text { (mean) } \\
\S \\
\end{array}$ & $415 / 323 \S$ & NGS/WGA & $4 / 703(0.6 \%)$ & $3 / 613(0.5 \%)$ & $1 / 90(1.1 \%)$ & $1 / 70(1.4 \%)$ \\
\hline Courville et al., 2013 [40] & $\begin{array}{l}\text { Retrospective, } \\
\text { multicenter }\end{array}$ & 44 & NA & NA & PCR & $2 / 44(4.5 \%)$ & - & $2 / 44(4.5 \%)$ & $2 / 44(4.5 \%)$ \\
\hline Itzykson et al., 2013 [64] & $\begin{array}{l}\text { Multicenter, } \\
\text { retrospective }\end{array}$ & 260 & $74(41-93)$ & $210 / 102$ & PCR & $3 / 260(1.1 \%)$ & - & $3 / 260(1.1 \%)$ & $3 / 260(1.1 \%)$ \\
\hline
\end{tabular}


Table 1. Cont.

\begin{tabular}{|c|c|c|c|c|c|c|c|c|c|}
\hline Reference & Type of Study & $\begin{array}{l}\text { Number of } \\
\text { Cases }\end{array}$ & $\begin{array}{l}\text { Median Age } \\
\text { (Range), } \\
\text { Years }\end{array}$ & $\operatorname{Sex}(M / F)$ & $\begin{array}{l}\text { Molecular } \\
\text { Analysis }\end{array}$ & $\begin{array}{l}\text { Overall Frequency of } \\
\text { NPM1 Mutations } \\
\text { Analyzed in MNs } \\
\text { with }<20 \% \text { Blasts }\end{array}$ & $\begin{array}{l}\text { Frequency of NPM1 } \\
\text { Mutations in } \\
\text { MDS Cases }\end{array}$ & $\begin{array}{l}\text { Frequency of } N P M 1 \\
\text { Mutations in } \\
\text { MDS/MPN Cases }\end{array}$ & $\begin{array}{l}\text { Frequency of NPM1 } \\
\text { Mutations in } \\
\text { CMML Cases }\end{array}$ \\
\hline Walter et al., 2013 [65] & $\begin{array}{l}\text { Monocenter, } \\
\text { retrospective }\end{array}$ & $157^{\wedge}$ & $\begin{array}{c}93 \mathrm{pt}(62 \%)> \\
60 \text { years }\end{array}$ & $92 / 58$ & NGS & $4 / 150(2.7 \%)$ & $4 / 150(2.7 \%)$ & - & - \\
\hline Wang et al., 2014 [66] & $\begin{array}{l}\text { Multicenter, } \\
\text { retrospective }\end{array}$ & 134 & $72(42-88) \S$ & $89 / 45 \S$ & PCR & $0 / 37$ & - & $0 / 37$ & - \\
\hline Haferlach et al., 2014 [67] & Multicenter & 944 & $\begin{array}{c}72.8 \\
(23.3-90.8) \\
\end{array}$ & $580 / 364$ & NGS & $27 / 944(2.9 \%)$ & $27 / 944(2.9 \%)$ & - & - \\
\hline Xu et al., 2014 [68] & Multicenter & 196 & 56 & $109 / 87$ & WGS/PCR & $5 / 196(2.6 \%)$ & $5 / 196(2.6 \%)$ & - & - \\
\hline Forghieri et al., 2015 [69] & $\begin{array}{l}\text { Monocenter, } \\
\text { retrospective }\end{array}$ & 177 & $77(47-93)$ & $109 / 68$ & PCR & $4 / 135(3 \%)$ & $2 / 108(1.9 \%)$ & $2 / 27(7.4 \%)$ & $0 / 16$ \\
\hline Cargo et al., 2015 [70] & $\begin{array}{l}\text { Monocenter, } \\
\text { retrospective }\end{array}$ & 69 & NA & NA & PCR/NGS & $3 / 69(4.3 \%)$ & $3 / 69(4.3 \%)$ & - & - \\
\hline Peng et al., 2016 [71] & $\begin{array}{l}\text { Monocenter, } \\
\text { retrospective }\end{array}$ & 152 & $72(27-92)$ & $110 / 42$ & PCR/NGS & $8 / 152(5.3 \%)$ & - & $8 / 152(5.3 \%)$ & $8 / 152(5.3 \%)$ \\
\hline Bartels et al., 2016 [72] & $\begin{array}{l}\text { Monocenter, } \\
\text { retrospective }\end{array}$ & $185 *(125) \S \S$ & $72(14-91)$ & NA & NGS & $1 / 81(1.2 \%) \S \S$ & $1 / 47(2.1 \%) \S \S$ & $0 / 44 \S \S$ & $0 / 34 \S \S$ \\
\hline Reinig et al., 2016 [73] & $\begin{array}{l}\text { Monocenter, } \\
\text { retrospective }\end{array}$ & $110^{*}$ & $63(5-83) \S$ & $77 / 33 \S$ & NGS & $0 / 53$ & $0 / 39$ & $0 / 14$ & $0 / 14$ \\
\hline Makishima et al., 2017 [74] & Multicenter & 699 & NA & NA & NGS & $32 / 1890(1.7 \%)^{\circ 0}$ & $32 / 1890(1.7 \%)^{\circ 0}$ & - & - \\
\hline Vallapureddy et al., 2017 [75] & $\begin{array}{l}\text { Monocenter, } \\
\text { retrospective }\end{array}$ & 373 & $71(20-95)$ & $246 / 127$ & NGS & $8 / 373(2 \%)$ & - & $8 / 373(2 \%)$ & $8 / 373(2 \%)$ \\
\hline Xu et al., 2017 [76] & $\begin{array}{l}\text { Monocenter, } \\
\text { prospective }\end{array}$ & 125 & $49(14-82)$ & $83 / 42$ & NGS & $2 / 125(1.6 \%)$ & $2 / 125(1.6 \%)$ & - & - \\
\hline Idossa et al., 2018 [77] & $\begin{array}{l}\text { Monocenter, } \\
\text { retrospective }\end{array}$ & 357 & $74(28-96)$ & $250 / 107$ & NGS & NA & NA & NA & NA \\
\hline Hamilton et al., 2018 [78] & $\begin{array}{l}\text { Multicenter, } \\
\text { retrospective }\end{array}$ & 80 & $52(12-70)$ & $43 / 37$ & NGS & $2 / 80(2.5 \%)$ & $2 / 80(2.5 \%)$ & - & - \\
\hline Montalban-Bravo et al., 2019 [79] & $\begin{array}{l}\text { Multicenter, } \\
\text { retrospective }\end{array}$ & 1900 & $62(19-86)^{\wedge}$ & $13 / 18^{\wedge}$ & PCR/NGS & $31 / 1900(1.6 \%)$ & NA & NA & NA \\
\hline Hwang et al., 2019 [80] & $\begin{array}{l}\text { Monocenter, } \\
\text { retrospective }\end{array}$ & 35 & $71(18-85)$ & $24 / 11$ & NGS & $4 / 35(11.4 \%)$ & - & $4 / 35(11.4 \%)$ & $4 / 35(11.4 \%)$ \\
\hline Zheng et al., 2019 [81] & $\begin{array}{l}\text { Monocenter, } \\
\text { prospective }\end{array}$ & $207^{* * *}$ & $60(4-91) \S$ & $113 / 94 \S$ & NGS & $2 / 115(1.7 \%)$ & $2 / 115(1.7 \%)$ & - & - \\
\hline Vantyghem et al., 2020 [82] & Multicenter & $177^{* * *}$ & $60(10-87) \S$ & $100 / 77 \S$ & NGS & $0 / 78$ & $0 / 40$ & $0 / 38$ & - \\
\hline
\end{tabular}


Table 1. Cont.

\begin{tabular}{|c|c|c|c|c|c|c|c|c|c|}
\hline Reference & Type of Study & $\begin{array}{l}\text { Number of } \\
\text { Cases }\end{array}$ & $\begin{array}{c}\text { Median Age } \\
\text { (Range), } \\
\text { Years }\end{array}$ & $\operatorname{Sex}(M / F)$ & $\begin{array}{l}\text { Molecular } \\
\text { Analysis }\end{array}$ & $\begin{array}{l}\text { Overall Frequency of } \\
N P M 1 \text { Mutations } \\
\text { Analyzed in MNs } \\
\text { with }<20 \% \text { Blasts }\end{array}$ & $\begin{array}{l}\text { Frequency of NPM1 } \\
\text { Mutations in } \\
\text { MDS Cases }\end{array}$ & $\begin{array}{l}\text { Frequency of NPM1 } \\
\text { Mutations in } \\
\text { MDS/MPN Cases }\end{array}$ & $\begin{array}{l}\text { Frequency of NPM1 } \\
\text { Mutations in } \\
\text { CMML Cases }\end{array}$ \\
\hline Wang et al., 2020 [83] & $\begin{array}{l}\text { Multicenter, } \\
\text { retrospective }\end{array}$ & $\begin{array}{c}406^{\wedge}(279 \\
\text { MDS cases) }\end{array}$ & $50(18-74)^{* * *}$ & $26 / 13^{* *}$ & NGS/PCR & $0 / 39^{* *}$ & $0 / 39 * *$ & - & - \\
\hline Badar et al., 2020 [44] & $\begin{array}{l}\text { Monocenter, } \\
\text { retrospective }\end{array}$ & $\begin{array}{c}646^{\wedge}(310 \\
\text { MDS cases) }\end{array}$ & $\begin{array}{l}72 \text { (MDS } \\
\text { cases) }\end{array}$ & NA & NGS & $4 / 263(1.5 \%)$ & $4 / 263(1.5 \%)$ & - & - \\
\hline Yu et al., 2020 [84] & $\begin{array}{l}\text { Monocenter, } \\
\text { retrospective }\end{array}$ & 93 & $46(16-87)$ & $56 / 37$ & NGS & $1 / 93(1.1 \%)$ & $1 / 93(1.1 \%)$ & - & - \\
\hline Wu et al., 2020 [85] & $\begin{array}{l}\text { Monocenter, } \\
\text { retrospective }\end{array}$ & 194 & $52(28-66)^{\wedge}$ & $7 / 5^{\wedge}$ & PCR/NGS & $12 / 194(6.2 \%)$ & $12 / 194(6.2 \%$ & - & - \\
\hline Yun et al., 2020 [86] & $\begin{array}{l}\text { Monocenter, } \\
\text { prospective }\end{array}$ & $\begin{array}{c}157 \text { (95 MDS, } \\
\text { CMML 10, } \\
\text { sAML 52 } \\
\text { cases) }\end{array}$ & $67(40-90) \S$ & $93 / 64 \S$ & NGS & $8 / 157(5 \%)^{\wedge}$ & - & - & - \\
\hline
\end{tabular}

MDS, myelodysplastic syndrome; MDS/MPN, myelodysplastic/myeloproliferative neoplasm; MNs, myeloid neoplasms; CMML, chronic myelomonocytic leukemia; NA; not available; PCR, polymerase chain reaction; NGS, next generation sequencing; WGA, whole genome amplification; WGS, whole genome sequencing; sAML, secondary acute myeloid leukemia. * The entire patient cohort also included AML and MPN cases. ${ }^{\wedge}$ The entire patient cohort included also AML cases. ${ }^{\circ}$ Only therapy-related MNs were examined in this series. $§$ Demographics refer to the entire patient cohort. ${ }^{* *}$ Results refer to 39 RUNX1-mutated MDS cases only ^^ Information provided refers to NPM1-mutated cases only $\$ \S$ Data refer to 125 patients who had at least one detectable pathogenic somatic mutations ${ }^{\circ 0}$ Results from previously published data sets are included in the final analysis ${ }^{* * *}$ The entire cohort also included patients with undefined cytopenia or aplastic anemia. 
Table 2. Clinical characteristics and prognostic features of NPM1-mutated MNs with $<20 \%$ blasts: review of the literature.

\begin{tabular}{|c|c|c|c|c|c|c|c|}
\hline Reference & $\begin{array}{c}\text { Number of } \\
\text { Patients/Median Age } \\
\text { (Range), Years }\end{array}$ & $\begin{array}{l}\text { Diagnosis According to } \\
\text { WHO Classification }\end{array}$ & WBC Count $\left(\times 10^{9} / \mathrm{L}\right)$ & $\begin{array}{l}\text { PB/BM Blast Count } \\
\text { (Median \%, Range) }\end{array}$ & Cytogenetics & IPSS/IPSS-R & Additional Molecular Lesions \\
\hline Caudill et al., 2006 [45] & $3 / \mathrm{NA}$ & CMML $3(100 \%)$ & $>12$ in one case & $\mathrm{NA} /<5 \%$ & NK $2(66.7 \%)$ & $\mathrm{NA}$ & FLT3 0 \\
\hline Oki et al., 2006 [46] & $2 / 78,77$ & CMML $2(100 \%)$ & $15,6.4$ & $\begin{array}{c}\mathrm{NA} / 14 \\
1 / 6\end{array}$ & NK $2(100 \%)$ & NA & NA \\
\hline Zhang et al., 2007 [47] & $2 / 40,67$ & $\begin{array}{l}\text { MDS-SLD } 1(50 \%) \\
\text { MDS-EB1 } 1(50 \%)\end{array}$ & $\begin{array}{l}3.8 \\
2.9\end{array}$ & $\mathrm{NA} / 1,6$ & 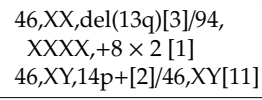 & 1] & NA \\
\hline Andersen et al., 2008 [50] § & $\begin{array}{l}3 \mathrm{t}-\mathrm{MN} / \\
69,39,35\end{array}$ & $\begin{array}{c}\text { MDS } 1(33.3 \%) \\
\text { MDS-SLD (RA) } 1(33.3 \%) \\
\text { MDS-SLD (RA) } 1(33.3 \%)\end{array}$ & NA & $\begin{array}{l}\text { NA/NA for } 1 \text { case, }<5 \% \\
\text { for the two RA cases }\end{array}$ & $\begin{array}{c}\text { NK } 1(33.3 \%) \\
\text { Complex } \\
\text { karyotype } 1 \\
(33.3 \%) \\
47, \mathrm{XX},+81 \text { case } \\
(33.3 \%)\end{array}$ & NA & $\begin{array}{l}\text { NRAS } 1(33.3 \%) \\
\text { FLT3 } 1(33.3 \%)\end{array}$ \\
\hline Ernst et al., 2010 [53] & 6/72 (53-77) & CMML $6(100 \%)$ & NA & NA & NA & NA & FLT3 0 \\
\hline Bejar et al., 2011 [59] & $8 / \mathrm{NA}^{\circ}$ & $\mathrm{NA}^{\circ}$ & $\mathrm{NA}^{\circ}$ & $\mathrm{NA} / \mathrm{NA}^{\circ}$ & NK $5(62.5 \%)$ & $\mathrm{NA} / \mathrm{NA}^{\circ}$ & TET2 $1(12.5 \%)$ \\
\hline Bains et al., 2011 [62] & $7 / 64(33-87)$ & $\begin{array}{c}\text { MDS 1 }(14.3 \%) \\
\text { MDS-EB 2 (28.6\%) } \\
\text { t-MN 1 (14.3\%) } \\
\text { CMML 2 (28.6\%) } \\
\text { MDS/MPN 1 (14.3\%) }\end{array}$ & NA & $\mathrm{NA} / 7(3-10)$ & NK $7(100 \%)$ & NA & $\begin{array}{c}\text { FLT3 } 4(57.1 \%) \\
\text { RAS 0 } \\
\text { KIT 0 } \\
\text { JAK2 } 0\end{array}$ \\
\hline Courville et al., 2013 [40] & $2 / 79,40$ & CMML $2(100 \%)$ & NA & NA & $\begin{array}{l}\text { NK } 1(50 \%) \\
\text { NA } 1 \text { case }\end{array}$ & NA & FLT3 2 (100\%) \\
\hline Forghieri et al., 2015 [69] & $4 / 68(47-85)$ & $\begin{array}{c}\text { aCML } 1(25 \%)^{* * *} \\
\text { MDS-MPN-u } 1(25 \%)^{* *} \\
\text { MDS-EB } 2(50 \%)^{\circ \circ}\end{array}$ & 11 (range, 1.3-24.9) & $\begin{array}{c}5(0-10) / \\
15(10-19)\end{array}$ & $\begin{array}{l}\text { NK } 3(75 \%) \\
\text { NA } 1(25 \%)\end{array}$ & $\mathrm{NA} / \mathrm{NA}$ & FLT3 0 \\
\hline Peng et al., 2016 [71] & $8 / 72(27-87)$ & $\begin{array}{c}\text { CMML-1 } 4(50 \%) \\
\text { CMML-2 } 4(50 \%) \\
\text { (proliferative type } \\
\text { CMML 6, 75\%) }\end{array}$ & 20 (range, 8.1-28.7) & $\begin{array}{l}1(0-3) / \\
2(0-14)\end{array}$ & NK $6(75 \%)$ & NA & $\begin{array}{c}\text { FLT3 0 } \\
\text { NRAS 2 (25\%) } \\
\text { DNMT3A (25\%) }\end{array}$ \\
\hline Vallapureddy et al., 2017 [75] & $8 / 76(48-87)$ & $\begin{array}{c}\text { Proliferative CMML } \\
\text { subtype 1 (13\%) } \\
\text { Dysplastic CMML subtype } \\
7(87 \%)\end{array}$ & 11 (range 3.7-186) & $\begin{array}{l}1(0-12) / \\
5(0-15)\end{array}$ & NK $8(100 \%)$ & $\begin{array}{l}\text { NA ( } 4,50 \% \text { high } \\
\text { risk according to } \\
\text { Mayo molecular } \\
\text { prognostic model) }\end{array}$ & $\begin{array}{c}\text { FLT3 } 1(13 \%) \\
\text { DNMT3A 4 (50\%) } \\
\text { NRAS } 2(25 \%) \\
\text { SRSF2 2 }(25 \%) \\
\text { TET2 } 1(13 \%)\end{array}$ \\
\hline
\end{tabular}


Table 2. Cont.

\begin{tabular}{|c|c|c|c|c|c|c|c|}
\hline Reference & $\begin{array}{c}\text { Number of } \\
\text { Patients/Median Age } \\
\text { (Range), Years }\end{array}$ & $\begin{array}{l}\text { Diagnosis According to } \\
\text { WHO Classification }\end{array}$ & WBC Count $\left(\times 10^{9} / \mathrm{L}\right)$ & $\begin{array}{l}\text { PB/BM Blast Count } \\
\text { (Median \%, Range) }\end{array}$ & Cytogenetics & IPSS/IPSS-R & Additional Molecular Lesions \\
\hline Montalban-Bravo et al., 2019 [79] & $31 / 62(19-86)$ & $\begin{array}{l}\text { MDS-EB } 19(62 \%) \\
\text { MDS del(5q) } 1(3 \%) \\
\text { MDS-MPN-U 2(6\%) } \\
\text { aCML 1 }(3 \%) \\
\text { CMML } 6(20 \%) \\
\text { t-MDS 2 (6\%) }\end{array}$ & 7.9 (range, $4.5-11.3$ ) & $\begin{array}{l}1(0-16) / \\
10(0-19)\end{array}$ & NK 24 (77\%) & $\begin{array}{c}\text { Int-2 + high } 29 \\
(94 \%) / \text { Intermediate } \\
13(42 \%), \text { high }+ \\
\text { very high } 18(58 \%)\end{array}$ & $\begin{array}{c}\text { FLT3 } 3(9.7 \%) \\
\text { NRAS } 7(22.6 \%) \\
\text { DNMT3A } 6(19.4 \%) \\
\text { TET2 } 4(12.9 \%) \\
\text { IDH2 } 2(6.5 \%)\end{array}$ \\
\hline Patel et al., 2019 [87] & $45 / 63(36-96)$ & $\begin{array}{c}\text { MDS non-EB } 2(4 \%) \\
\text { MDS-EB } 24(54 \%) \\
\text { CMML } 9(20 \%) \\
\text { MDS/MPN } 5(11 \%) \\
\text { t-MN } 5(11 \%)\end{array}$ & 3.3 (range, $1.2-225$ ) & $\begin{array}{c}\mathrm{NA} / \\
10(1-19)\end{array}$ & NK $40(89 \%)$ & $\begin{array}{c}\text { NA/median score } \\
\text { for MDS cases only } \\
5 \text { (range } 1.5-7 \text { ) }\end{array}$ & $\begin{array}{c}\text { DNMT3A } 15(33.3 \%) \\
\text { IDH1/2 6(13\%) } \\
\text { TET2 } 7(16 \%) \\
\text { FLT3 4 }(8.9 \%) \\
\text { NRAS } 4(8.9 \%) \\
\text { SF3B1 4 }(8.9 \%) \\
\end{array}$ \\
\hline Hwang et al., 2019 [80] & $4 / 71(18-85) *$ & $\begin{array}{c}\text { CMML-0 } 15(42.9 \%) \\
\text { CMML-1 } 7(20 \%) \\
\text { CMML-2 } 13(37.1 \%) \\
\text { (proliferative subtype 28, } \\
80 \%)^{*}\end{array}$ & $19.4($ range, $4.6-141)$ * & $\mathrm{NA} / \mathrm{NA}$ & NK $25(71-4)$ * & NA & $\begin{array}{c}\text { DNMT3A } 4(100 \%) \\
\text { FLT3 } 1(25 \%) \\
\text { TET2 } 1(25 \%)\end{array}$ \\
\hline Wu et al., 2020 [85] & $12 / 52(28-66)$ & $\begin{array}{l}\text { MDS-EB } 9(75 \%) \\
\text { CMML } 2(25 \%)\end{array}$ & NA & NA/15 (4-19) & NK $9(75 \%)$ & $\begin{array}{c}\text { Int-2 + high 7 } \\
(58.3 \%) / \text { Intermediate } \\
2(16.7 \%) \text {, high + } \\
\text { very high 10 } \\
(83.3 \%)\end{array}$ & $\begin{array}{c}\text { DNMT3A } 6(50 \%) \\
\text { IDH2 } 2(16.7 \%) \\
\text { FLT3 1 }(8.3 \%)\end{array}$ \\
\hline
\end{tabular}

MNs, myeloid neoplasms; WHO, World Health Organization; WBC, white blood cell; PB, peripheral blood; BM, bone marrow; IPSS, international prognostic scoring system; IPSS-R, international prognostic scoring system-revised; NA, not available; CMML, chronic myelomonocytic leukemia; NK, normal karyotype; MDS, myelodysplastic syndrome; MDS-SLD, myelodysplastic syndrome with single lineage dysplasia; MDS-EB, myelodysplastic syndrome with excess blasts; MDS-MPN; myelodysplastic syndrome-myeloproliferative neoplasm; aCML; atypical $B C R-A B L 1$ negative chronic myeloid leukemia, IHC, immunohistochemical; AML; acute myeloid leukemia. * Clinical characteristics refer to the whole 35 patient cohort. ${ }^{\circ}$ No detailed clinical information on selected NPM1-mutated cases is available. $§$ Only therapy-related MNs were examined in this series. ${ }^{* *}$ In these two cases, which were initially presumptively diagnosed, upon morphologic analysis, as aCML and MDS/MPN-U, respectively, NPMc+ AML was finally documented, based upon molecular assays on BM aspirate and presumptively diagnosed, upon morphologic analysis, as aCML and MDS/MPN-U, respectively, NPMc+ AML was finally documented, based upon molecular assays on BM aspirate and
IHC examinations on BM trephine biopsy. ${ }^{\circ \circ}$ The IHC examinations, retrospectively performed on BM trephine biopsies from these two elderly deceased subjects, documented extensive IHC examinations on BM trephine biopsy. ${ }^{\circ}$ The IHC examinations, retrospectively performed on BM trephine biopsies from these two elderly deceased subjects, documented extensive
NPMc+ staining with multilineage involvement, suggesting that NPM1-mutated AML, rather than MDS, would have been presumptively diagnosed, ab initio, in both cases by the NPMc+ staining with multilineage involvement,
combination of molecular and IHC investigations. 


\section{Clinical and Genetic Features of NPM1-Mutated MNs with $<20 \%$ Blasts}

Since the earliest observations, the rare finding of NPM1 mutations in MNs with $<20 \%$ blasts in small and mainly retrospective series, has been commonly associated with an aggressive clinical course and relatively rapid progression to overt AML, usually within 12 months since diagnosis, as detailed in Table $3[5,40,45,46,62,69,71,75]$. However, most of these previous studies have interrogated for NPM1 mutations using limited single-gene PCR assays, without the possibility to compare the genetic profiles of NPM1-mutated MNs with the mutational landscape of most frequent MDS and MDS/MPN cases lacking NPM1 mutations [87,88]. Several studies examining large numbers of MDS and CMML samples by high-throughput sequencing technologies at diagnosis have identified more than $40-50$ recurrently mutated genes, with greater than $80-90 \%$ of patients showing at least one somatic gene mutation (Table 4) $[59,63,67,89-96]$. Of note, it is widely recognized that approximately $30 \%$ and $15-20 \%$ of patients initially observed for either MDS or CMML, respectively, eventually experience AML transformation, with variable incidence according to age, genetic characteristics and prognostic stratification scores $[89,90,93,95,97-101]$. 
Table 3. Therapeutic approaches and clinical outcomes of NPM1-mutated MNs with $<20 \%$ blasts: review of the literature.

\begin{tabular}{|c|c|c|c|c|c|c|c|c|}
\hline Reference & Intensive CHT & HMAs & ORR/CR Rates (\%) & Allogeneic HSCT & $\begin{array}{l}\text { Median Follow-Up } \\
\text { Time (Months) }\end{array}$ & Progression to AML & Time to Progression (Months) & Survival Outcomes \\
\hline Caudill et al., 2006 [45] & NA & NA & NA/NA & NA & NA & 3 cases $(100 \%)$ & Within 12 months & 8 months \\
\hline Oki et al., 2006 [46] & 0 & $\begin{array}{c}1(50 \%) \\
\text { decitabine }\end{array}$ & $\begin{array}{c}\text { NA/1 CR with } \\
\text { decitabine }\end{array}$ & 0 & NA & $\begin{array}{c}1 \text { previously } \\
\text { untreated case }(50 \%)\end{array}$ & 12 months & NA \\
\hline Zhang et al., 2007 [47] & NA & NA & NA/NA & NA & NA & NA & NA & $\begin{array}{l}24 \text { months/lost of } \\
\text { follow-up }\end{array}$ \\
\hline Andersen et al., 2008 [50] § & NA & NA & NA/NA & NA & $\mathrm{NA}$ & 2 cases $(66.7 \%)$ & $\begin{array}{l}20 \text { months/ } \\
16 \text { months }\end{array}$ & NA \\
\hline Ernst et al., 2010 [53] & $\mathrm{NA}^{\circ}$ & $\mathrm{NA}^{\circ}$ & $\mathrm{NA} / \mathrm{NA}^{\circ}$ & $\mathrm{NA}^{\circ}$ & $\mathrm{NA}^{\circ}$ & $\mathrm{NA}^{\circ}$ & $\mathrm{NA}^{\circ}$ & $\begin{array}{l}\text { Median PFS and OS } \\
<24 \text { months }\end{array}$ \\
\hline Bejar et al., 2011 [59] & $\mathrm{NA}^{\circ}$ & $\mathrm{NA}^{\circ}$ & $\mathrm{NA} / \mathrm{NA}{ }^{\circ}$ & $\mathrm{NA}^{\circ}$ & $\mathrm{NA}^{\circ}$ & $\mathrm{NA}^{\circ}$ & $\mathrm{NA}^{\circ}$ & $\begin{array}{l}\text { Median survival } \\
26 \text { months }\end{array}$ \\
\hline Bains et al., 2011 [62] & $\begin{array}{c}1(14.3 \%) \\
\text { clofarabine and } \\
\text { cytarabine }\end{array}$ & $\begin{array}{c}3(42.3 \%) \\
\text { decitabine }\end{array}$ & NA/NA & 0 & 7-14 months & $\begin{array}{c}4 \text { FLT3-mutated cases } \\
(57.1 \%)\end{array}$ & $12(2-13)$ & $\begin{array}{c}\text { FLT3 in combination } \\
\text { with NPM1 mutations } \\
\text { had a significant } \\
\text { negative impact } \\
\text { on PFS }\end{array}$ \\
\hline Courville et al., 2013 [40] & $\mathrm{NA}^{\circ}$ & $\mathrm{NA}^{\circ}$ & $\mathrm{NA} / \mathrm{NA}^{\circ}$ & $\mathrm{NA}^{\circ}$ & $\mathrm{NA}^{\circ}$ & 2 cases $(100 \%)$ & $\begin{array}{l}3 \text { months/ } \\
0.5 \text { months }\end{array}$ & $\begin{array}{l}\text { Dead } 12 \text { months and } \\
\text { alive } 11 \text { months, } \\
\text { respectively, since } \\
\text { AML diagnosis }\end{array}$ \\
\hline Forghieri et al., 2015 [69] & $2(50 \%)^{*}$ & $1(25 \%)$ & $\begin{array}{c}2 \mathrm{CR}(100 \%) \text { in cases } \\
\text { treated with } \\
\text { induction CHT * }\end{array}$ & $1(25 \%)$ & 50 (range, 2-121) & $\begin{array}{c}1 \text { case, after having } \\
\text { received } 6 \text { cycles of } \\
5-\mathrm{AZA}\end{array}$ & $\begin{array}{c}6 \text { months (after } 6 \text { cycles of } \\
\text { 5-AZA) }\end{array}$ & $\begin{array}{l}2 \text { patients alive at } 121 \\
\text { and } 90 \text { months, } \\
\text { respectively, since } \\
\text { NPM1-mutated AML } \\
\text { diagnosis }{ }^{*} \\
2 \text { patients died } 12 \text { and } \\
2 \text { months, } \\
\text { respectively, since } \\
\text { MDS diagnosis }{ }^{\circ}\end{array}$ \\
\hline Peng et al., 2015 [71] & $4(50 \%)$ & $5(62.5 \%)$ & NA/NA & $2(25 \%)$ & NA & 4 cases $(50 \%)$ & 11 (range, 1-21) & $\begin{array}{c}5 \text { patients died at } 5 \text { to } \\
34 \text { months } \\
\text { since diagnosis }\end{array}$ \\
\hline Vallapureddy et al., 2017 [75] & $\begin{array}{l}4 \text { out of } 5 \text { cases, } \\
\text { at AML } \\
\text { transformation }\end{array}$ & 0 & NA/NA & $\begin{array}{l}2 \text { cases after AML } \\
\text { transformation }\end{array}$ & 9.4 (range, $0.3-41)$ & 5 cases $(63 \%)$ & 5 (range, 1-16) & $\begin{array}{c}\text { Median DFS } 9 \text { months } \\
\text { and OS } 12.5 \text { months }\end{array}$ \\
\hline
\end{tabular}


Table 3. Cont.

\begin{tabular}{|c|c|c|c|c|c|c|c|c|}
\hline Reference & Intensive CHT & HMAs & ORR/CR Rates (\%) & Allogeneic HSCT & $\begin{array}{l}\text { Median Follow-Up } \\
\text { Time (Months) }\end{array}$ & $\begin{array}{l}\text { Progression to } \\
\text { AML }\end{array}$ & Time to Progression (Months) & Survival Outcomes \\
\hline Montalban-Bravo et al., 2019 [79] & $10(32 \%)$ & $20(65 \%)$ & $\begin{array}{c}100 \% \text { with IC, } 83 \% \\
\text { with HMAs/ } 90 \% \\
\text { with IC, } 28 \% \text { with } \\
\text { HMAs }\end{array}$ & $13(42 \%)$ & 17.6 (range, $1-106$ ) & 12 cases $(38.7 \%)$ & 14 (range, 7-34) & $\begin{array}{c}\text { Globally, median OS } \\
25.7 \text { months. With IC, } \\
\text { median OS NR and } \\
\text { PFS NR; with HMAs, } \\
\text { median OS } 16 \text { months } \\
\text { and PFS } 7.5 \text { months }\end{array}$ \\
\hline Patel et al., 2019 [87] & $3(7 \%)$ & $33(73 \%)$ & NA/NA & $19(42 \%)$ & 10 (range, $0.07-70$ ) & 20 cases $(44 \%)$ & 5.2 (range, $0.4-17.5$ ) & Median OS 20 months \\
\hline Hwang et al., 2019 [80] & $3(11 \%)^{* *}$ & $16(45.7 \%)^{* *}$ & $37.5 \% / 18.8 \% * *$ & $5(14.3 \%)^{* *}$ & 16.8 (range, $0.1-101$ ) ** & $\begin{array}{c}10(28.6 \%)^{* *} \\
2 / 4 \text { cases }(50 \%)\end{array}$ & range $7.4-9.6$ & $\begin{array}{c}\text { Median OS } 21.5 \\
\text { months } \text { N }^{*} \text { No } \\
\text { significant difference } \\
\text { in OS and PFS } \\
\text { between patients with } \\
\text { or without } \\
\text { NPM1 mutations }\end{array}$ \\
\hline Wu et al., 2020 [85] & 0 & $\begin{array}{l}12(100 \%) \\
\text { decitabine }\end{array}$ & $\begin{array}{c}66.7 \% / 50 \%(83.3 \% \text { in } \\
\text { cases with } \\
\text { DNMT3A WT) }\end{array}$ & $1(8.3 \%)$ & NA & NA & NA & $\begin{array}{c}\text { Median RFS of CR } \\
\text { cases and OS of } \\
\text { patients without } \\
D N M T 3 A \text { mutations } \\
66 \text { and } 80 \\
\text { months, respectively. }\end{array}$ \\
\hline
\end{tabular}

MNs, myeloid neoplasms; CHT, chemotherapy, HMA, hypomethylating agents; ORR, overall response rate; CR, complete remission; HSCT, hematopoietic stem cell transplantation; AML, acute myeloid leukemia; NA; not available; PFS, progression-free survival; OS, overall survival; DFS, disease-free survival; MDS, myelodysplastic syndrome; IC, intensive chemotherapy; NR, not reached; 5-AZA, 5-azacytidine; RFS, relapse-free survival..$^{\circ}$ No detailed clinical information on selected NPM1-mutated cases is available. § Only therapy-related MNs were examined in this series. * In these two cases, which were initially presumptively diagnosed, upon morphologic analysis, as aCML and MDS/MPN-U, respectively, NPMc+ AML was finally documented, based upon molecular assays on BM aspirate and IHC examinations on BM trephine biopsy. ${ }^{\circ}$ The IHC examinations, retrospectively performed on BM trephine biopsies from these two elderly deceased subjects, documented extensive NPMc+ staining with multilineage involvement, suggesting that NPM1-mutated AML, rather than MDS, would have been presumptively diagnosed, ab initio, in both cases by the combination of molecular and IHC investigations. ${ }^{* *}$ Clinical characteristics refer to the whole 35 patient cohort. 
Table 4. Overall incidence of cytogenetic/molecular lesions in MDS and CMML patients at diagnosis.

\begin{tabular}{|c|c|c|c|}
\hline & & $\begin{array}{c}\text { Overall Frequency in } \\
\text { MDS Cases }(\%) \S\end{array}$ & $\begin{array}{l}\text { Overall Frequency in } \\
\text { CMML Cases }(\%) \S\end{array}$ \\
\hline \multicolumn{2}{|c|}{$\begin{array}{l}\text { Clonal Cytogenetic Abnormalities by Metaphase } \\
\text { Karyotyping }\end{array}$} & $50-60$ & $10-40$ \\
\hline \multicolumn{4}{|c|}{ Recurrently Mutated Genes } \\
\hline \multirow[t]{7}{*}{ Epigenetic regulators } & TET2 & $20-25$ * & $30-60^{\wedge}$ \\
\hline & ASXL1 & $5-25 *$ & $40-50^{\wedge}$ \\
\hline & DNMT3A & $2-18$ * & $2-12$ \\
\hline & EZH2 & $5-10$ & $5-12$ \\
\hline & IDH1 & $<5$ & $1-2$ \\
\hline & IDH2 & $<5$ & $6-7$ \\
\hline & $B C O R$ & $<5$ & $5-10$ \\
\hline \multirow[t]{4}{*}{ Spliceosome } & SF3B1 & $20-30$ * & $5-10$ \\
\hline & SRSF2 & $10-15$ * & $30-50^{\wedge}$ \\
\hline & $U 2 A F 1$ & $8-12$ & $5-10$ \\
\hline & ZRSR2 & $5-10$ & $5-10$ \\
\hline \multirow{6}{*}{ Signal transduction } & NRAS & $5-10$ & $10-20$ \\
\hline & KRAS & $5-10$ & $10-20$ \\
\hline & $C B L$ & $<5$ & $10-20$ \\
\hline & $N F 1$ & $<5$ & $5-10$ \\
\hline & $J A K 2$ & $<5$ & $1-10$ \\
\hline & FLT3 & $<5$ & $1-3$ \\
\hline \multirow[t]{2}{*}{$\begin{array}{l}\text { DNA damage/Cell cycle } \\
\text { regulators }\end{array}$} & TP53 & $8-12$ & $<5$ \\
\hline & PHF6 & $<5$ & $<5$ \\
\hline Chromosome topology & $\begin{array}{l}\text { Cohesin complex (mostly } \\
\text { STAG2) }\end{array}$ & $5-10$ & $5-10$ \\
\hline \multirow[t]{4}{*}{ Transcription factors } & RUNX1 & $10-15$ & $10-30$ \\
\hline & SETBP1 & $<5$ & $5-20$ \\
\hline & ETV6 & 2 & $<1$ \\
\hline & NPM1 & 2 & 3 \\
\hline
\end{tabular}

MDS, myelodysplastic syndrome; CMML, chronic myelomonocytic leukemia. § A median of three gene mutations (range 0-17) per case are found in MDS patients, while an average of 10-15 somatic mutations can be detected in CMML patients. * Most frequent gene mutations documented in MDS cases at diagnosis. ^ Most frequently observed gene mutations in CMML patients at diagnosis.

Bains et al. documented FLT3 and NPM1 mutations in $2 \%$ and $4.4 \%$ of cases, respectively, from a large cohort of non-acute MNs, with NPM1 mutations being significantly associated with normal karyotype and higher risk MDS or MDS/MPN [62]. However, none of the three patients with NPM1 mutation alone progressed to AML after 7-14 months follow-up, while the four subjects with concurrent FLT3 mutations invariably developed AML with a median interval of 12 months, with independent negative impact on progression-free survival (PFS) [62]. Based upon these preliminary data, the authors inferred that NPM1 mutations alone could not be adequate to explain progression of MDS to AML, requiring further genetic lesions, such as FLT3 gene mutations [62]. However, while the two NPM1-mutated CMML cases described by Courville et al., rapidly evolving to AML, also disclosed FLT3 mutations [40], several further patients with NPM1-mutated non-acute MN diagnosis, experienced AML progression despite the absence of concurrent FLT3 mutations $[71,75,79,80,87]$. In the series by Peng et al., a comparison with 144 CMML patients without NPM1 mutations documented that NPM1-mutated cases presented more severe anemia, higher BM monocyte percentage, an increased tendency to AML progression and shorter OS, although these two latter comparisons did not reach statistical significance [71]. None of the 8 NPM1-mutated CMML patients had FLT3 mutations, either at diagnosis or during the course of the disease. Moreover, frequency of mutations involving NRAS/KRAS, TET2 and ASXL1 were not significantly different between the two patient groups. Of interest, four subjects (50\%), all with high NPM1 mutation allele burden $>10 \%$, developed AML, suggesting that NPM1 mutation alone, if at low level, could not have a direct role in AML progression and may need additional genetic lesions to induce disease transformation [71]. Accordingly, in the study by Vallapureddy et al., NPM1-mutated CMML patients were more likely to be anemic and to have a dysplastic CMML phenotype, compared with NPM1 wild-type counterparts, while an increased incidence of DNMT3A and FLT3 mutations and a lower frequency of TET2 and ASXL1 mutations 
were observed in NPM1-mutated subgroups [75]. Furthermore, the eight CMML cases harboring NPM1 mutations showed aggressive clinical behavior with higher risk of blast transformation (63\%), occurring at a median of 5 months since initial diagnosis, compared to patients with NPM1 wild-type $(18 \%)$. In multivariate analysis, leukemia-free survival (LFS) was significantly adversely influenced by higher PB blast percentage, TP53 and NPM1 mutations [75]. Patel et al. have recently described clinical and biological data of 45 patients with NPM1-mutated MN with $<20 \%$ blasts, the largest multicenter cohort so far collected [87]. Information from additional cohorts of NPM1 wild-type MNs and de novo NPM1-mutated AML was evaluated for comparison. Compared with NPM1 wild-type MNs, NPM1-mutated MNs were associated with younger patient age, a normal karyotype, more frequent mutations involving DNMT3A and PTPN11, but harbored fewer mutations in ASXL1, RUNX1 and TP53 genes. Moreover, in comparison with AML showing NPM1 mutations, NPM1-mutated MNs exhibited significantly fewer mutations in IDH1, IDH2 and FLT3 genes, and a trend toward fewer gene mutations involving NRAS and KRAS. Most patients with NPM1-mutated MNs (73\%) received upfront treatment with hypomethylating agents (HMA) and thirteen of them (39\%) progressed to AML at a median time of 5.2 months, while none of the cases receiving intensive induction chemotherapy experienced leukemic evolution. Interestingly, in this study no statistically significant difference in rate of leukemic evolution and time to transformation could be established between NPM1-mutated and unmutated MNs cohorts. However, based on five NPM1-mutated MNs cases who did not receive any upfront therapy and subsequently experienced early AML progression after a median observation of three months, the authors inferred that the upfront HMA treatment received by the majority of NPM1-mutated MNs cases could have favorably altered the clinical course of the underlying myeloid malignancy, therefore delaying a potentially more rapid AML transformation. However, poor clinical outcomes were collectively observed for NPM1-mutated MNs, with shorter median OS (20 months), as compared with those documented for NPM1 wild-type MNs (36.6 months) and NPM1-mutated AML (42.2 months) cases. In multivariable analysis performed only in 86 patients with a diagnosis of MDS, including 26 NPM1-mutated cases, total mutation count, presence of TP53 or NPM1 mutations, higher IPSS-R score were factors independently associated with shorter OS, whereas HSCT conferred a favorable effect. It could thus be suggested that non-acute NPM1-mutated MNs, characterized by aggressive clinical behavior, could benefit from more intensive therapeutic approaches [87]. Consistent with this, similar findings were reported in parallel by Montalban-Bravo et al. in a smaller patient series [79]. In details, the 31 patients affected with NPM1-mutated MNs, mainly MDS-EB (61\% of cases), were younger, had lower hemoglobin levels, had higher median BM blast percentage at diagnosis and showed a higher frequency of normal karyotype, compared with NPM1 wild-type patients. All the cases showed multilineage dysplasia at diagnosis on morphologic examinations. Of interest, while there was no correlation between NPM1 mutational burden and BM or PB blast percentage at baseline, mutation clearance at time of $\mathrm{CR}$ after therapy was associated with clearance of trilineage dysplastic features. Relevant to this, cases treated upfront with intensive chemotherapy obtained significantly higher CR rates, longer PFS and OS, compared with patients who received HMA, as detailed in Table 3. Moreover, a total of 13 patients, namely, seven treated with intensive chemotherapy and six after HMA, underwent allogeneic HSCT, collectively resulting in favorable survival outcomes compared to cases not receiving HSCT (median OS, not reached versus 22.1 months, $p=0.012$ ). Although it should be noted that the sample size is small, the analysis among different treatment subgroups showed that HSCT was associated with significantly prolonged survival only in patients treated with HMA (2-year OS, $67 \%$ versus $28 \%, p=0.025$ ), whereas no improvement in survival was documented for subjects receiving intensive chemotherapy (2-year OS, 80\% versus 100\%, $p=0.655$ ). In summary, although the median OS of 25.7 months globally recorded in this patient cohort was certainly unsatisfactory, intensive treatment approaches, including remission induction chemotherapy and HSCT, for fit patients with NPM1-mutated MNs may correlate with improved clinical outcomes [79]. Conversely, Wu et al. recently identified a specific small subgroup of MDS patients harboring NPM1 mutations with DNMT3A wild-type, with particularly favorable outcomes after decitabine therapy [85]. 
Among a total of 194 MDS cases receiving upfront therapy with decitabine $20 \mathrm{mg} / \mathrm{m}^{2}$ for 5 consecutive days every 4-6 weeks, NPM1-mutated MDS patients achieved a relatively higher CR rate (6 of 12 cases, $50 \%$ ), compared to cases with NPM1 wild-type (53 of 182 cases, $29.1 \%$ ). Furthermore, patients harboring NPM1 mutation in the absence of DNMT3A mutations obtained a CR rate of $83.3 \%$ ( 5 of 6 cases), which was significantly higher than that of MDS patients without NPM1 mutations. A significantly longer RFS period was observed in NPM1-mutated and DNMT3A wild-type MDS patients obtaining $\mathrm{CR}$, even without any different subsequent therapy. Finally, a markedly prolonged median OS was also documented in MDS genetic subgroup with NPM1 mutations and DNMT3A wild-type, compared with cases negative for NPM1 mutations (80 versus 18 months, $p=0.012$ ). Except for DNMT3A and PTPRD co-mutations, the response to treatment and favorable survival outcomes of this small NPM1-mutated MDS patient cohort, were not negatively influenced by co-mutations in IDH2, NRAS and FLT3 genes, highlighting the importance of identifying molecular landscapes, predictive of response to different therapeutic approaches [85]. By targeted gene sequencing on BM samples from 35 Korean patients with CMML, Hwang et al. recently found slightly different mutational profiles, with lower frequency of TET2 mutations (25.7\%) and higher frequencies of DNMT3A $(17.1 \%), N R A S(31.4 \%)$ and NPM1 $(11.4 \%)$ mutations compared with those documented in previous studies from Caucasian CMML cases as summarized in Table 4 [80]. These differences could be related to the observation of a small number of CMML patients or alternatively to different occurrence of somatic mutations among ethnicities. Moreover, in this latter study, no significant differences were observed in rate of leukemic transformation or in survival outcomes between CMML patients with or without NPM1 mutations, probably due to the limited number of patients analyzed [80]. In summary, to the best of our knowledge, detailed information about therapeutic approaches in non-acute NPM1-mutated MNs are available for only 27 and 75 patients receiving intensive chemotherapy and HMA, respectively, as shown in Table 2. Due to the limited number of available data, mainly from retrospective studies lacking a controlled clinical trial design, no firm evidence-based conclusion can so far be drawn about the best treatment for NPM1-mutated MNs with $<20 \%$ blasts $[5,79,87,88]$. Nevertheless, overall poor outcomes have been observed in most NPM1-mutated MNs patients, and upfront moderate intensity therapy, based on HMA, could be frequently considered inadequate. On the contrary, NPM1-mutated MNs patients who are fit and candidate for undergoing more intensive treatments, potentially including allogeneic HSCT, may have improved survival outcomes compared with historical data, and could therefore benefit most from remission induction chemotherapy, rather than from MDS-directed therapeutic approaches, despite the presence of $<20 \%$ blasts $[5,79,87]$. Falini et al. also tend to treat patients affected with NPM1-mutated MDS or CMML according to the same therapeutic recommendations provided for NPM1-mutated AML [5]. However, prospective multicenter clinical trials are needed to further investigate these controversial issues.

\section{Pathological Classification of NPM1-Mutated MNs with < $20 \%$ Blasts: A Controversial Issue}

Another relevant subject of controversy is represented by the pathological classification of these uncommon cases of MNs showing NPM1 gene mutations with $<20 \%$ circulating and BM blast counts, also with potentially significant implications on the choice of best therapeutic approaches $[5,69]$. While the documentation of recurrent cytogenetic/molecular abnormalities, namely $\mathrm{t}(15 ; 17)$ (q22;q12), $\mathrm{t}(8 ; 21)(\mathrm{q} 22 ; \mathrm{q} 22)$ or inv(16)(p13.1q22)/t(16;16)(p13.1;q22), is actually recognized to be sufficient, according to the WHO-2016 classification, to define a diagnosis of AML, independently of the blast percentage, the finding of NPM1 gene mutation is not currently considered to be sufficient to diagnose NPM1-mutated AML in cases with $<20 \%$ blasts $[5,21,69]$. However, a diagnostic dilemma may be raised for the interpretation of the clinico-pathologic significance of NPM1 mutations in the context of MDS or MDS/MPN cases, since multilineage involvement and dysplastic features are frequently exhibited in NPM1-mutated AML [2,5,21,48,69,102-104]. Of note, Falini et al. previously documented, in a large series of 318 NPM1-mutated AML patients, that multilineage dysplasia, detected in approximately $23 \%$ of cases, had no significant impact on gene expression profile or pathologic, immunophenotypic, 
clinical, and prognostic features of NPM1-mutated AML. These findings preliminarily indicated that the observation of NPM1 mutation should predominate over multilineage dysplasia as disease-defining criterion [103]. Accordingly, due to the lack of prognostic significance of multilineage dysplasia in patients without MDS-associated cytogenetic findings and with a mutation of NPM1 or biallelic mutation of CEBPA, the revised WHO 2016 classification of myeloid neoplasms defined that these genetic lesions now supersede the morphological presence of multilineage dysplasia in the diagnostic classification [21]. Therefore, NPM1-mutated AML showing multilineage dysplasia should be distinguished from MDS-related changes AML [5,21]. Based upon the observation by Pasqualucci et al. that NPMc+ immunohistochemical pattern may detect clonal multilineage involvement in NPM1-mutated AML [102] and that limited and inconclusive information had previously been reported on immunohistochemical examinations to investigate sub-cellular localization of NPM1 protein in non-acute MNs $[12,40,49,69]$, we have hypothesized that in rare cases of either MDS or MDS/MPN with a blast count invariably $<20 \%$, but showing NPM1 mutation on molecular assays, AML may be under-diagnosed [69]. We have thus suggested, by an integrated molecular and immunohistochemical diagnostic approach, that the documentation of extensive NPMc+ staining in more than $20 \%$ of cells with multilineage involvement, on BM trephine biopsies of two adult patients with presumptive morphological diagnosis of MDS/MPN but harboring NPM1 mutations, could be sufficient to eventually define a NPMc+ AML diagnosis [69], as detailed in Table 2. Accordingly, these two latter patients achieved CR after remission induction chemotherapy, and subsequently received consolidation with either autologous (patient 1) or allogeneic (patient 2) HSCT, experiencing favorable clinical outcomes, as shown in Table 3 [69]. Based upon these preliminary observations, we have retrospectively analyzed a cohort of further 175 adult patients affected with either MDS or MDS/MPN from our Institution [69]. By including the previously mentioned patients 1 and 2 with MDS/MPN, BM aspirate samples were available for screening the presence of exon-12 NPM1 mutations by qualitative PCR analysis in 135 cases (Table 1). NPM1 mutations were retrospectively tracked in two elderly patients, formerly diagnosed with MDS-EB2, who accordingly received 5-azacytidine and best supportive care, respectively (Tables 2 and 3). Of interest, the immunohistochemical staining, retrospectively performed on BM trephine biopsies even from these two deceased subjects, documented diffuse NPMc + staining in $>20 \% \mathrm{BM}$ cells with multilineage involvement, therefore suggesting that NPMc+ AML, rather than MDS, could have been presumptively diagnosed, $a b$ initio, in both these cases by the combination of NPM1 molecular and immunohistochemical investigations [69]. While neither FLT3-ITD nor FLT3-TKD mutations were observed, the presence of additional molecular lesions was unfortunately not investigated by high-throughput sequencing tools in our four cases with normal karyotype. However, it should be noted that a distinct gene expression profile characterized by up-regulation of HOX and MEIS1 genes and lower expression of CD34, resembling that associated with NPM1-mutated AML, was invariably identified in our patients (Paolini A. et al., personal observation). Even if we acknowledge that the blast proportion should be enumerated in BM aspirate and cannot generally be extrapolated by immunohistochemical examinations, except for cases of dry tap $[10,69,95]$, we have considered that immunohistochemical reaction for NPM1 protein, carried out on BM trephine biopsy, when NPM1 mutation is detected by PCR analysis in patients with MDS or MDS/MPN, may interestingly allow to precisely evaluate multilineage BM cells with NPMc+ staining, belonging to the NPM1-mutated leukemic clone regardless of blast morphology $[69,102]$. Furthermore, in the experience of Falini et al. BM trephine biopsies from NPM1-mutated MDS or CMML often show clusters of NPMc+ blasts, suggestive of early AML [5]. Future investigations are warranted to precisely define whether the documentation of NPM1 gene mutation per se may become sufficient, in the appropriate clinical setting, to classify MNs as NPM1-mutated AML, independently of the blast cell count [5,69]. This topic should certainly be a matter of debate for the next revision of WHO classification of myeloid neoplasms [5]. 


\section{Clonal Hematopoiesis, NPM1 Mutations and Cooperating Molecular Lesions in Promoting Leukemogenesis: A Lesson from Mouse Models.}

Cheng et al. first described in 2010 in vivo evidence that NPM1 mutations could confer a proliferative advantage in the mature granulocytic/monocytic lineage of transgenic mice expressing NPM1 mutation under the myeloid-specific $h M R P 8$ promoter [105]. Notably, the non-reactive myeloproliferation found in BM and spleen from $h M R P 8-N P M c+$ transgenic mice did not progress to overt leukemia, perhaps because the NPMc+ transgenic model did not exactly reproduce the NPM1-mutated human AML cell expression pattern, suggesting the need for cooperating mutations [105]. Further subsequent genetically engineered mouse models of NPM1 mutation, including transgenic and knock-in alleles, allowed the generation of mice with a constant genotype and a reproducible phenotype. These mouse models of NPM1-mutated AML have certainly been important for demonstrating that the NPM1 mutation alone, though inducing deregulated cell growth, displayed a low leukemogenic activity in vivo, but can lead to leukemia progression after long latency and acquisition of collaborating mutations [106]. In more details, Guryanova et al. recently reported the lack of overt AML development in Npm1cA1+/Dnmt3aR878H compound model, whereas Npm1/Flt3-ITD/Dnmt3a triple-mutated mice invariable succumbed from a particularly aggressive AML, confirming the role of co-mutated genes in dictating whether leukemogenesis does occur or not in murine Npm1-mutated models [107]. Other groups also reported that Npm1/Flt3-ITD double mutated mice generated a fully penetrant and short latency AML [108,109]. Furthermore, Dovey et al. interestingly compared the effects of double-mutated genotypes, namely the combination of NPM1 mutation with either FLT3-ITD or NRAS lesions, on hematopoiesis and leukemogenesis in knock-in mice [110]. Npm1cA/1;NrasG12D/1 or Npm1cA;Flt3-ITD compound genotypes shared a number of consequences on hematopoiesis, namely Hox gene over-expression, higher self-renewal capacity, expansion of hematopoietic progenitors, and myeloid differentiation bias. However, Npm1cA;Flt3-ITD mutants displayed more aggressive behavior, significantly higher peripheral white blood cell counts and a monocytic differentiation in comparison with the granulocytic bias observed in Npm1cA/1;NrasG12D/1 mutants. Moreover, while both double-mutant models developed high-penetrance AML, latency was significantly longer with Npm1cA/1;NrasG12D/1 [110,111]. Finally, in mice genetically engineered through a dual-recombinase system, Loberg et al. recently described the sequential induction of Dnmt3a mutation, leading to features resembling human clonal hematopoiesis, such as expansion of hematopoietic stem and multipotent progenitor cell compartments, and subsequent induction of mutant Npm1, which then caused progression of clonal hematopoiesis to a myeloproliferative disorder (MPD). Moreover, mice uniformly experienced AML progression from MPD following successive transplants. At a molecular level, progression of clonal hematopoiesis to MPD was accompanied by mutations activating Ras/Raf/MAPK signaling, while transformation to AML was characterized by additional oncogenic signaling mutations, namely in Ptpn11, Pik3r1, Flt3 genes and/or mutations in epigenetic regulators, such as Hdac1, Idh1, Arid1a [112]. In summary, NPM1 mutations could be found in preleukemic settings in mouse models, usually with either myeloproliferative or myelodysplastic features, and may act as a marker of progression to AML. Intriguingly, Uckelmann et al. described the possibility to eradicate preleukemic NPM1-mutated proliferating and self-renewing myeloid progenitors, using targeted epigenetic therapy, namely VTP-50469, an inhibitor of Menin-MLL1 interaction, with the achievement of differentiation and growth arrest [113]. Indeed, the authors showed that early intervention targeting chromatin regulators and therefore preventing the occurrence of full-blown AML, is possible in a Npm1/Dnmt3a mutant conditional knock-in mouse model, and suggested that similar preventative epigenetic approaches could become a future possibility also for humans at high risk of developing AML [113]. Although relevant to investigate distinct preleukemic Npm1-mutated populations, it should be noted that these multistep leukemogenesis mouse models are hardly reproducible in humans, where NPM1 mutations are not associated with clonal hematopoiesis of indeterminate potential (CHIP) and are overall infrequently documented in MDS or MDS/MPN cases, as reported above and summarized in Tables 1 and 4, 
while almost invariably correlated to an AML diagnosis [114,115]. In more details, unlike mutations in genes involved in chromatin remodeling, namely DNMT3A, TET2 and ASXL1, or RNA splicing, such as SF3B1 and SRSF2, NPM1 mutations are not detectable in individuals with CHIP [5,115]. A further note of caution about widely tracking occurrence of preleukemic clones and their subsequent targeting in humans should be raised, because there are no current indications to screening for CHIP in the general population $[114,115]$. An actually accepted model of clonal evolution in humans, also supported by studies at single cell level, suggests that NPM1 mutations may be a secondary later event, acting as a "gatekeeper" in the pathogenesis of NPM1-mutated AML, and occurring in the setting of clonal hematopoiesis, characterized by founder mutations mainly involving DNA methylation pathway-related genes $[5,88,115-120]$. Of interest, NPM1-mutated AML patients who obtain long-term MRD-negative CR, but returning to a clonal hematopoiesis status, e.g., with persistent DNMT3A gene mutation, could be predisposed to development of a second different myeloid neoplasm. Therefore, although most relapses in NPM1-mutated AML patients are due to the reappearance of the original NPM1-mutated clone, nearly $5-10 \%$ of AML recurrences are characterized by the absence of NPM1 mutations, preferably suggesting that a second de novo or therapy-related AML with NPM1 wild-type could raise from persistent clonal hematopoiesis, after the eradication of the original NPM1-mutated AML clone [5,18,121-123]. In summary, while in preclinical models NPM1-mutation has widely been identified as a significant transforming event, which contributes to leukemogenesis, but generally insufficient alone to drive full-blown AML, distinct clonal NPM1-mutated preleukemic populations cannot be found in humans, where the occurrence of NPM1 mutation may largely be considered an AML-defining event [5,123]. Accordingly, since VTP-50469 and MI-3454 also showed efficacy against NPM1-mutated AML in patient-derived xenograft assays [113,124], further investigations on potential benefit of inhibition of Menin-MLL chromatin complex in patients with frank leukemia or, alternatively to pre-emptively target leukemia-specific NPM1-mutated clones in the setting of persisting MRD, are warranted [5,114]. In the future, targeting HOX expression through Menin-MLL inhibition could thus potentially add to other non-chemotherapic agents, such as dactinomycin or venetoclax, which have recently shown promising anti-leukemic activity in distinct subgroups of NPM1-mutated AML patients [125-127].

\section{Conclusions}

Caution is needed in definitely diagnose NPM1-mutated MNs with blast count $<20 \%$, since NPM1-mutated AML cases frequently present multilineage involvement and dysplastic features on morphologic and immunohistochemical grounds [2,5,69,102]. Moreover, the rare cases of NPM1-mutated MNs, mainly belonging to high-risk MDS and CMML, usually show normal karyotype, negativity for CD34 expression on blasts and aggressive clinical behavior with relatively rapid progression to overt AML, raising controversies on their classification as distinct clinico-pathologic entities $[5,69,71,75,79,87,88]$. Relevant to this, relatively favorable treatment responses are observed when these patients receive intensive chemotherapy rather than HMA, further resembling AML clinical behavior $[5,69,79,87]$. Based upon these observations, it could be suggested to routinely investigate the presence of NPM1 mutations by molecular techniques, in MDS and MDS/MPN, at least in higher risk cases with normal karyotype. Subsequently, immunohistochemical examinations on BM trephine biopsy should be carried out in cases harboring NPM1 mutations, thus allowing an integrated molecular and immunohistochemical diagnostic approach [69]. Interestingly, Itzykson et al. observed that, although not yet considered an AML-defining lesion, the presence of NPM1 mutation in CMML tends to be associated to an aggressive clinical course, suggesting that finding NPM1 mutations may favor a diagnosis of de novo AML exhibiting dysplastic features and monocytic differentiation, belonging to M4 or M5 AML subgroups according to former FAB classification, rather than CMML [90]. Of note, upgrading a case of higher risk MDS or MDS/MPN to a definitive NPM1-mutated AML diagnosis, based upon molecular and/or immunohistochemical analyses, could have relevant consequences on therapeutic algorithm. In details, for younger and fit patients affected with higher risk MDS or CMML, 
allogeneic HSCT is generally recommended, either upfront if BM blast count is less than $10 \%$ or after cytoreductive treatment in cases with blast percentage $>10 \%$. Moreover, standard induction chemotherapy could usually be suggested as a bridge to transplant cytoreduction for patients with favorable or intermediate risk karyotype, whereas HMA may be preferred for cases showing unfavorable genetic lesions $[89,100]$. Conversely, patients diagnosed with NPM1-mutated AML, especially when belonging to more favorable risk subgroups according to ELN classification [10], could potentially benefit from intensive remission induction and consolidation chemotherapeutic approaches only, without firm indication to receive upfront allogeneic HSCT, unless for cases with FLT3-ITD positivity, relapsed/refractory disease or persistently elevated MRD levels [20]. From a molecular point of view, it is widely recognized that progression from MDS to AML is generally associated with an increased mutation burden, in terms of number of variants and/or VAFs [128]. The assessment and monitoring of genetic abnormalities could provide a measure of tumour burden that often greatly exceeds the BM blast percentage. Indeed, in MDS patients molecular lesions could be documented by modern high-throughput platforms in most BM cells, regardless of the blast count [98]. Therefore, using the blast count to define a precise boundary between MDS and AML secondary to MDS may have limitations, because disease progression is considered as a continuum [98]. Of note, in both NPM1-mutated AML and NPM1-mutated MNs, immunohistochemistry could easily detect multilineage BM cells with NPMc+ staining, belonging to the NPM1-mutated clone regardless of blast morphology $[69,106,129]$. Interestingly, the NCCN guidelines for MDS recently allowed the classification of patients having $20 \%$ to $29 \%$ BM blasts as MDS-EB in transformation (MDS-EB-T) rather than AML, a definition carried over from the former FAB classification, but the authors also observed that individuals carrying NPM1 and/or FLT3 mutations are more likely to have AML than MDS [89]. In conclusion, prospective multicenter studies on larger patient cohorts are warranted to further assess biological and clinical features of NPM1-mutated MNs with blast count $<20 \%$, and to definitely investigate whether the observation of NPM1 gene mutations may become sufficient to define AML, irrespective of blast percentage found in $\mathrm{PB}$ or $\mathrm{BM}$ samples, as already established in the cases of core-binding factor AML harboring either RUNX1-RUNX1T1 or CBFbeta-MYH11 fusion transcripts [5,10,21,69].

Author Contributions: F.F., V.N. and A.P. designed the study, reviewed the literature and wrote the manuscript; F.B., V.P., D.G., A.G., C.C., G.A., G.R., P.B., R.M. (Rossana Maffei) and L.P. reviewed the literature and wrote the manuscript; R.M. (Roberto Marasca), C.F., E.T., T.T., P.C., G.L. and M.L. supervised the study, analyzed data and critically revised the manuscript. All authors have read and agreed to the published version of the manuscript.

Funding: This research received no external funding.

Acknowledgments: This work was supported by grants from the Associazione Italiana per la Ricerca sul Cancro (AIRC), Milan, Italy (IG 20624-2018) (ML), the Progetto di Eccellenza Dipartimento MIUR 2017 (ML), and the "Charity Dinner initiative" in memory of Alberto Fontana for Associazione Italiana Lotta alle Leucemie, Linfoma e Mieloma (AIL) —Sezione 'Luciano Pavarotti'-Modena-ONLUS. This work was also supported by grants from the Fondazione Regionale per la Ricerca Biomedica (PC); Fondazione Istituto di Ricovero e Cura a Carattere Scientifico (IRCCS) Policlinico San Matteo di Pavia [Ricerca Corrente 08069113 and 08069119 (PC)]; Fondazione Just Italia (PC).

Conflicts of Interest: Authors' disclosures of potential conflict of interest. F.F. served on advisory boards for Jannsen on the clinical use of decitabine, for Novartis on the clinical use of midostaurin and eltrombopag and received travel grants from Jazz Pharmaceuticals. M.L. served on advisory boards for Novartis on the clinical use of midostaurin, for AbbVie, on the clinical use of venetoclax, for Jazz Pharmaceuticals, on the clinical use of Vyxeos, for Gilead Sci., on the clinical use of Ambisome, for MSD, on the clinical use of letermovir, for Sanofi, on the clinical use of caplacizumab, from Daiichi-Sankyo, for the clinical use of quizartinib and received travel grants from Gilead Sci and Sanofi. The other authors declare no potential conflicts of interest.

\section{References}

1. Grisendi, S.; Mecucci, C.; Falini, B.; Pandolfi, P.P. Nucleophosmin and cancer. Nat. Rev. Cancer 2006, 6, 493-505. [CrossRef] [PubMed]

2. Falini, B.; Nicoletti, I.; Martelli, M.F.; Mecucci, C. Acute myeloid leukemia carrying cytoplasmic/mutated nucleophosmin (NPMc + AML): Biologic and clinical features. Blood 2007, 109, 874-885. [CrossRef] [PubMed] 
3. Falini, B.; Bolli, N.; Liso, A.; Martelli, M.P.; Mannucci, R.; Pileri, S.; Nicoletti, I. Altered nucleophosmin transport in acute myeloid leukaemia with mutated NPM1: Molecular basis and clinical implications. Leukemia 2009, 23, 1731-1743. [CrossRef] [PubMed]

4. Falini, B.; Martelli, M.P.; Bolli, N.; Sportoletti, P.; Liso, A.; Tiacci, E.; Haferlach, T. Acute myeloid leukemia with mutated nucleophosmin (NPM1): Is it a distinct entity? Blood 2011, 117, 1109-1120. [CrossRef]

5. Falini, B.; Brunetti, L.; Sportoletti, P.; Martelli, M.P. NPM1-mutated acute myeloid leukemia: From bench to bedside. Blood 2020, 136, 1707-1721. [CrossRef]

6. Riback, J.A.; Zhu, L.; Ferrolino, M.C.; Tolbert, M.; Mitrea, D.M.; Sanders, D.W.; Wei, M.T.; Kriwacki, R.W.; Brangwynne, C.P. Composition-dependent thermodynamics of intracellular phase separation. Nature 2020, 581, 209-214. [CrossRef]

7. Mitrea, D.M.; Cika, J.A.; Stanley, C.B.; Nourse, A.; Onuchic, P.L.; Banerjee, P.R.; Phillips, A.H.; Park, C.G.; Deniz, A.A.; Kriwacki, R.W. Self-interaction of NPM1 modulates multiple mechanisms of liquid-liquid phase separation. Nat. Commun. 2018, 9, 842. [CrossRef]

8. Nachmani, D.; Bothmer, A.H.; Grisendi, S.; Mele, A.; Bothmer, D.; Lee, J.D.; Monteleone, E.; Cheng, K.; Zhang, Y.; Bester, A.C.; et al. Germline NPM1 mutations lead to altered rRNA 2'-Omethylation and cause dyskeratosis congenita. Nat. Genet 2019, 51, 1518-1529. [CrossRef]

9. Grimwade, D.; Ivey, A.; Huntly, B.J.P. Molecular landscape of acute myeloid leukemia in younger adults and its clinical relevance. Blood 2016, 127, 29-41. [CrossRef]

10. Dohner, H.; Estey, E.; Grimwade, D.; Amadori, S.; Appelbaum, F.R.; Buchner, T.; Dombret, H.; Ebert, B.L.; Fenaux, P.; Larson, R.A.; et al. Diagnosis and management of AML in adults: 2017 ELN recommendations from an international expert panel. Blood 2017, 129, 424-447. [CrossRef]

11. Papaemmanuil, E.; Gerstung, M.; Bullinger, L.; Gaidzik, V.I.; Paschka, P.; Roberts, N.D.; Potter, N.E.; Heuser, M.; Thol, F.; Bolli, N.; et al. Genomic classification and prognosis in acute myeloid leukemia. N. Engl. J. Med. 2016, 374, 2209-2221. [CrossRef] [PubMed]

12. Falini, B.; Mecucci, C.; Tiacci, E.; Alcalay, M.; Rosati, R.; Pasqualucci, L.; La Starza, R.; Diverio, D.; Colombo, E.; Santucci, A.; et al. Cytoplasmic nucleophosmin in acute myelogenous leukemia with a normal karyotype. $N$. Engl. J. Med. 2005, 352, 254-266. [CrossRef] [PubMed]

13. Heath, E.M.; Chan, S.M.; Minden, M.D.; Murphy, T.; Shlush, L.I.; Schimmer, A.D. Biological and clinical consequences of NPM1 mutations in AML. Leukemia 2017, 31, 798-807. [CrossRef] [PubMed]

14. Borrow, J.; Dyer, S.A.; Akiki, S.; Griffiths, M.J. Molecular roulette: Nucleophosmin mutations in AML are orchestrated through N-nucleotide addition by TdT. Blood 2019, 134, 2291-2303. [CrossRef]

15. Falini, B.; Martelli, M.P.; Bolli, N.; Bonasso, R.; Ghia, E.; Pallotta, M.T.; Diverio, D.; Nicoletti, I.; Pacini, R.; Tabarrini, A.; et al. Immunohistochemistry predicts nucleophosmin (NPM) mutations in acute myeloid leukemia. Blood 2006, 108, 1999-2005. [CrossRef]

16. Gu, X.; Ebrahem, Q.; Mahfouz, R.Z.; Hasipek, M.; Enane, F.; Radivoyevitch, T.; Rapin, N.; Przychodzen, B.; $\mathrm{Hu}, \mathrm{Z}$; Balusu, R.; et al. Leukemogenic nucleophosmin mutation disrupts the transcription factor hub regulating granulo-monocytic fates. J. Clin. Investig. 2018, 128, 4260-4279. [CrossRef]

17. Brunetti, L.; Gundry, M.C.; Sorcini, D.; Guzman, A.G.; Huang, Y.H.; Ramabadran, R.; Gionfriddo, I.; Mezzasoma, F.; Milano, F.; Nabet, B.; et al. Mutant NPM1 maintains the leukemic state through HOX expression. Cancer Cell 2018, 34, 499-512. [CrossRef]

18. Uckelmann, H.J.; Armstrong, S.A.; Stone, R.M. Location, location, location: Mutant NPM1c cytoplasmic localization is required to maintain stem cell genes in AML. Cancer Cell 2018, 34, 355-357. [CrossRef]

19. Höllein, A.; Meggendorfer, M.; Dicker, F.; Jeromin, S.; Nadarajah, N.; Kern, W.; Haferlach, C.; Haferlach, T. NPM1 mutated AML can relapse with wild-type NPM1: Persistent clonal hematopoiesis can drive relapse. Blood Adv. 2018, 2, 3118-3125. [CrossRef]

20. Forghieri, F.; Comoli, P.; Marasca, R.; Potenza, L.; Luppi, M. Minimal/Measurable Residual Disease monitoring in NPM1-mutated acute myeloid leukemia: A clinical viewpoint and perspectives. Int. J. Mol. Sci 2018, 19, 3492. [CrossRef]

21. Arber, D.A.; Orazi, A.; Hasserjian, R.; Thiele, J.; Borowitz, M.J.; Le Beau, M.M.; Bloomfield, C.D.; Cazzola, M.; Vardiman, J.W. The 2016 revision to the World Health Organization classification of myeloid neoplasms and acute leukemia. Blood 2016, 127, 2391-2405. [CrossRef] 
22. Haferlach, C.; Mecucci, C.; Schnittger, S.; Kohlmann, A.; Mancini, M.; Cuneo, A.; Testoni, N.; Rege-Cambrin, G.; Santucci, A.; Vignetti, M.; et al. AML with mutated NPM1 carrying a normal or aberrant karyotype show overlapping biologic, pathologic, immunophenotypic, and prognostic features. Blood 2009, 114, 3024-3032. [CrossRef]

23. Micol, J.B.; Boissel, N.; Renneville, A.; Castaigne, S.; Gardin, C.; Preudhomme, C.; Dombret, H. The role of cytogenetic abnormalities in acute myeloid leukemia with NPM1 mutations and no FLT3 internal tandem duplication. Blood 2009, 114, 4601-4602. [CrossRef]

24. Angenendt, L.; Röllig, C.; Montesinos, P.; Martínez-Cuadrón, D.; Barragan, E.; García, R.; Botella, C.; Martínez, P.; Ravandi, F.; Kadia, T.; et al. Chromosomal abnormalities and prognosis in NPM1-mutated acute myeloid leukemia: A pooled analysis of individual patient data from nine international cohorts. J. Clin. Oncol. 2019, 37, 2632-2642. [CrossRef]

25. Döhner, K.; Schlenk, R.F.; Habdank, M.; Scholl, C.; Rücker, F.G.; Corbacioglu, A.; Bullinger, L.; Fröhling, S.; Döhner, H. Mutant nucleophosmin (NPM1) predicts favorable prognosis in younger adults with acute myeloid leukemia and normal cytogenetics: Interaction with other gene mutations. Blood 2005, 106, 3740-3746. [CrossRef]

26. Gale, R.E.; Green, C.; Allen, C.; Mead, A.J.; Burnett, A.K.; Hills, R.K.; Linch, D.C.; Medical Research Council Adult Leukaemia Working Party. The impact of FLT3 internal tandem duplication mutant level, number, size, and interaction with NPM1 mutations in a large cohort of young adult patients with acute myeloid leukemia. Blood 2008, 111, 2776-2784. [CrossRef]

27. Pratcorona, M.; Brunet, S.; Nomdedéu, J.; Ribera, J.M.; Tormo, M.; Duarte, R.; Escoda, L.; Guàrdia, R.; Queipo de Llano, M.P.; Salamero, O.; et al. Favorable outcome of patients with acute myeloid leukemia harboring a low-allelic burden FLT3-ITD mutation and concomitant NPM1 mutation: Relevance to postremission therapy. Blood 2013, 121, 2734-2738. [CrossRef] [PubMed]

28. Schlenk, R.F.; Kayser, S.; Bullinger, L.; Kobbe, G.; Casper, J.; Ringhoffer, M.; Held, G.; Brossart, P.; Lübbert, M.; Salih, H.R.; et al. Differential impact of allelic ratio and insertion site in FLT3-ITD positive AML with respect to allogeneic transplantation. Blood 2014, 124, 3441-3449. [CrossRef] [PubMed]

29. Linch, D.C.; Hills, R.K.; Burnett, A.K.; Khwaja, A.; Gale, R.E. Impact of FLT3(ITD) mutant allele level on relapse risk in intermediate-risk acute myeloid leukemia. Blood 2014, 124, 273-276. [CrossRef]

30. Straube, J.; Ling, V.Y.; Hill, G.R.; Lane, S.W. The impact of age, NPM1(mut), and FLT3(ITD) allelic ratio in patients with acute myeloid leukemia. Blood 2018, 131, 1148-1153. [CrossRef] [PubMed]

31. Sakaguchi, M.; Yamaguchi, H.; Najima, Y.; Usuki, K.; Ueki, T.; Oh, I.; Mori, S.; Kawata, E.; Uoshima, N.; Kobayashi, Y.; et al. Prognostic impact of low allelic ratio FLT3-ITD and NPM1 mutation in acute myeloid leukemia. Blood Adv. 2018, 2, 2744-2754. [CrossRef] [PubMed]

32. Boddu, P.C.; Kadia, T.M.; Garcia-Manero, G.; Cortes, J.; Alfayez, M.; Borthakur, G.; Konopleva, M.; Jabbour, E.J.; Daver, N.G.; DiNardo, C.D.; et al. Validation of the 2017 European LeukemiaNet classification for acute myeloid leukemia with NPM1 and FLT3-internal tandem duplication genotypes. Cancer 2019, 125, 1091-1100. [CrossRef] [PubMed]

33. Döhner, K.; Thiede, C.; Jahn, N.; Panina, E.; Gambietz, A.; Larson, R.A.; Prior, T.W.; Marcucci, G.; Jones, D.; Krauter, J.; et al. Impact of NPM1/FLT3-ITD genotypes defined by the2017 European LeukemiaNet in patients with acute myeloid leukemia. Blood 2020, 135, 371-380. [CrossRef] [PubMed]

34. Eisfeld, A.K.; Kohlschmidt, J.; Mims, A.; Nicolet, D.; Walker, C.J.; Blachly, J.S.; Carroll, A.J.; Papaioannou, D.; Kolitz, J.E.; Powell, B.E.; et al. Additional gene mutations may refine the 2017 European LeukemiaNet classification in adult patients with de novo acute myeloid leukemia aged $<60$ years. Leukemia 2020. [CrossRef]

35. Schuurhuis, G.J.; Heuser, M.; Freeman, S.; Béné, M.C.; Buccisano, F.; Cloos, J.; Grimwade, D.; Haferlach, T.; Hills, R.K.; Hourigan, C.S.; et al. Minimal/measurable residual disease in AML: A consensus document from the European LeukemiaNet MRD Working Party. Blood 2018, 131, 1275-1291. [CrossRef]

36. Falini, B.; Martelli, M.P.; Pileri, S.A.; Mecucci, C. Molecular and alternative methods for diagnosis of acute myeloid leukemia with mutated NPM1: Flexibility may help. Haematologica 2010, 95, 529-534. [CrossRef]

37. Dicker, F.; Haferlach, C.; Sundermann, J.; Wendland, N.; Weiss, T.; Kern, W.; Haferlach, T.; Schnittger, S. Mutation analysis for RUNX1, MLL-PTD, FLT3-ITD, NPM1 and NRAS in 269 patients with MDS or secondary AML. Leukemia 2010, 24, 1528-1532. [CrossRef] [PubMed] 
38. Schnittger, S.; Bacher, U.; Haferlach, C.; Alpermann, T.; Dicker, F.; Sundermann, J.; Kern, W.; Haferlach, T. Characterization of NPM1-mutated AML with a history of myelodysplastic syndromes or myeloproliferative neoplasms. Leukemia 2011, 25, 615-621. [CrossRef]

39. Schnittger, S.; Haferlach, C.; Nadarajah, N.; Alpermann, T.; Meggendorfer, M.; Perglerova, K.; Kern, W.; Haferlach, T. In AML secondary to MDS NPM1 mutations are late events, less frequent, and associated with a different pattern of molecular mutations than in de novo AML. Blood 2014, 124, 700. [CrossRef]

40. Courville, E.L.; Wu, Y.; Kourda, J.; Roth, C.G.; Brockmann, J.; Muzikansky, A.; Fathi, A.T.; de Leval, L.; Orazi, A.; Hasserjian, R.P. Clinicopathologic analysis of acute myeloid leukemia arising from chronic myelomonocytic leukemia. Mod. Pathol. 2013, 26, 751-761. [CrossRef]

41. Fernandez-Mercado, M.; Yip, B.H.; Pellagatti, A.; Davies, C.; Larrayoz, M.J.; Kondo, T.; Pérez, C.; Killick, S.; McDonald, E.J.; Odero, M.D.; et al. Mutation patterns of 16 genes in primary and secondary acute myeloid leukemia (AML) with normal cytogenetics. PLoS ONE 2012, 7, e42334. [CrossRef]

42. Lindsley, R.C.; Mar, B.G.; Mazzola, E.; Grauman, P.V.; Shareef, S.; Allen, S.L.; Pigneux, A.; Wetzler, M.; Stuart, R.K.; Erba, H.P.; et al. Acute myeloid leukemia ontogeny is defined by distinct somatic mutations. Blood 2015, 125, 1367-1376. [CrossRef]

43. Wang, S.Y.; Cheng, W.Y.; Mao, Y.F.; Zhu, Y.M.; Liu, F.J.; Ma, T.T.; Shen, Y. Genetic alteration patterns and clinical outcomes of elderly and secondary acute myeloid leukemia. Hematol. Oncol. 2019, 35, 456-463. [CrossRef]

44. Badar, T.; Szabo, A.; Sallman, D.; Komrojki, R.; Lancet, J.; Padron, E.; Song, J.; Hussaini, M.O. Interrogation of molecular profiles can help in differentiating between MDS and AML with MDS-related changes. Leuk. Lymphoma 2020, 61, 1418-1427. [CrossRef]

45. Caudill, J.S.C.; Sternberg, A.J.; Li, C.Y.; Tefferi, A.; Lasho, T.L.; Steensma, D.P. C-terminal nucleophosmin mutations are uncommon in chronic myeloid disorders. Br. J. Haematol. 2006, 133, 638-641. [CrossRef]

46. Oki, Y.; Jelinek, J.; Beran, M.; Verstovsek, S.; Kantarjian, H.M.; Issa, J.P.J. Mutations and promoter methylation status of NPM1 in myeloproliferative disorders. Haematologica 2006, 91, 1147-1148.

47. Zhang, Y.; Zhang, M.; Yang, L.; Xiao, Z. NPM1 mutations in myelodysplastic syndromes and acute myeloid leukemia with normal karyotype. Leuk. Res. 2007, 31, 109-111. [CrossRef]

48. Shiseki, M.; Kitagawa, Y.; Wang, Y.H.; Yoshinaga, K.; Kondo, T.; Kuroiwa, H.; Okada, M.; Mori, N.; Motoji, T. Lack of nucleophosmin mutation in patients with myelodysplastic syndrome and acute myeloid leukemia with chromosome 5 abnormalities. Leuk. Lymphoma 2007, 48, 2141-2144. [CrossRef]

49. Ishikawa, Y.; Xu, J.; Sakashita, G.; Urano, T.; Suzuki, T.; Tomita, A.; Kiyoi, H.; Nakamura, S.; Naoe, T. Abnormal cytoplasmic dyslocation and/or reduction of nucleophosmin protein level rarely occurs in myelodysplastic syndromes. Leuk. Lymphoma 2008, 49, 2359-2364. [CrossRef]

50. Andersen, M.T.; Andersen, M.K.; Christiansen, D.H.; Pedersen-Bjergaard, J. NPM1 mutations in therapy-related acute myeloid leukemia with uncharacteristic features. Leukemia 2008, 22, 951-955. [CrossRef]

51. Bacher, U.; Haferlach, T.; Kern, W.; Weiss, T.; Schnittger, S.; Haferlach, C. The impact of cytomorphology, cytogenetics, molecular genetics, and immunophenotyping in a comprehensive diagnostic workup of myelodysplastic syndromes. Cancer 2009, 115, 4524-4532. [CrossRef] [PubMed]

52. Chen, W.; Huang, Q. Detection of FLT3/ITD, JAK2(V617F) and NPM1 gene mutations in chronic myelomonocytic leukemia. Leuk. Res. 2009, 33, e207-e209. [CrossRef] [PubMed]

53. Ernst, T.; Chase, A.; Zoi, K.; Waghorn, K.; Hidalgo-Curtis, C.; Score, J.; Jones, A.; Grand, F.; Reiter, A.; Hochhaus, A.; et al. Transcription factor mutations in myelodysplastic/myeloproliferative neoplasms. Haematologica 2010, 95, 1473-1480. [CrossRef] [PubMed]

54. Rocquain, J.; Carbuccia, N.; Trouplin, V.; Raynaud, S.; Murati, A.; Nezri, M.; Tadrist, Z.; Olschwang, S.; Vey, N.; Birnbaum, D.; et al. Combined mutations of ASXL1, CBL, FLT3, IDH1, IDH2, JAK2, KRAS, NPM1, NRAS, RUNX1, TET2 and WT1 genes in myelodysplastic syndromes and acute myeloid leukemias. BMC Cancer 2010, 10, 401. [CrossRef] [PubMed]

55. Li, L.; Zhang, Y.; Ma, X.T.; Yang, L.; Xu, Z.F.; Xiao, Z.J. Study of NPM1 gene mutations in patients with primary myelodysplastic syndromes. Zhonghua Xue Ye Xue Za Zhi 2010, 31, 809-812. [PubMed]

56. Thol, F.; Weissinger, E.M.; Krauter, J.; Wagner, K.; Damm, F.; Wichmann, M.; Göhring, G.; Schumann, C.; Bug, G.; Ottmann, O.; et al. IDH1 mutations in patients with myelodysplastic syndromes are associated with an unfavorable prognosis. Haematologica 2010, 95, 1668-1674. [CrossRef] 
57. Gritsaev, S.V.; Martynkevich, I.S.; Ivanova, M.P.; Moskalenko, M.V.; Aksenova, V.; Tiranova, S.A.; Abdulkadyrov, K.M. Investigation of FLT3-ITD and NPM1 mutations in patients with myelodysplastic syndrome and mixed myeloid diseases. Vopr. Onkol. 2010, 56, 671-676.

58. Gelsi-Boyer, V.; Trouplin, V.; Roquain, J.; Adélaïde, J.; Carbuccia, N.; Esterni, B.; Finetti, P.; Murati, A.; Arnoulet, C.; Zerazhi, H.; et al. ASXL1 mutation is associated with poor prognosis and acute transformation in chronic myelomonocytic leukaemia. Br. J. Haematol. 2010, 151, 365-375. [CrossRef]

59. Bejar, R.; Stevenson, K.; Abdel-Wahab, O.; Galili, N.; Nilsson, B.; Garcia-Manero, G.; Kantarjian, H.; Raza, A.; Levine, R.L.; Neuberg, D.; et al. Clinical effects of point mutations in myelodyplastic syndromes. N. Engl. J. Med. 2011, 364, 2496-2506. [CrossRef]

60. Bacher, U.; Haferlach, C.; Alpermann, T.; Kern, W.; Schnittger, S.; Haferlach, T. Comparison of genetic and clinical aspects in patients with acute myeloid leukemia and myelodysplastic syndromes all with more than $50 \%$ of bone marrow erythropoietic cells. Haematologica 2011, 96, 1284-1292. [CrossRef]

61. Machado-Neto, J.A.; Traina, F.; Lazarini, M.; Campos Pde, M.; Pagnano, K.B.; Lorand-Metze, I.; Costa, F.F.; Saad, S.T. Screening of hotspot mutations in PI3K, JAK2, FLT3 and NPM1 in patients with myelodysplastic syndromes. Clinics 2011, 66, 793-799. [PubMed]

62. Bains, A.; Luthra, R.; Medeiros, J.; Zuo, Z. FLT3 and NPM1 mutations in myelodysplastic syndromes. Frequency and potential value for predicting progression to acute myeloid leukemia. Am. J. Clin. Pathol. 2011, 135, 62-69. [CrossRef] [PubMed]

63. Papaemmanuil, E.; Gerstung, M.; Malcovati, L.; Tauro, S.; Gundem, G.; Van Loo, P.; Yoon, C.J.; Ellis, P.; Wedge, D.C.; Pellagatti, A.; et al. Clinical and biological implications of driver mutations in myelodysplastic syndromes. Blood 2013, 122, 3616-3627. [CrossRef]

64. Itzykson, R.; Kosmider, O.; Renneville, A.; Gelsi-Boyer, V.; Meggendorfer, M.; Morabito, M.; Berthon, C.; Adès, L.; Fenaux, P.; Beyne-Rauzy, O.; et al. Prognostic score including gene mutations in chronic myelomonocytic leukemia. J. Clin. Oncol. 2013, 31, 2428-2436. [CrossRef] [PubMed]

65. Walter, M.J.; Shen, D.; Shao, J.; Ding, L.; White, B.S.; Kandoth, C.; Miller, C.A.; Niu, B.; McLellan, M.D.; Dees, N.D.; et al. Clonal diversity of recurrently mutated genes in myelodysplastic syndromes. Leukemia 2013, 27, 1275-1282. [CrossRef]

66. Wang, S.A.; Hasserjian, R.P.; Fox, P.S.; Rogers, H.J.; Geyer, J.T.; Chabot-Richards, D.; Weinzierl, E.; Hatem, J.; Jaso, J.; Kanagal-Shamanna, R.; et al. Atypical chronic myeloid leukemia is clinically distinct from unclassifiable myelodysplastic/myeloproliferative neoplasms. Blood 2014, 123, 2645-2651. [CrossRef]

67. Haferlach, T.; Nagata, Y.; Grossmann, V.; Okuno, Y.; Bacher, U.; Nagae, G.; Schnittger, S.; Sanada, M.; Kon, A.; Alpermann, T.; et al. Landscape of genetic lesions in 944 patients with myelodysplastic syndromes. Leukemia 2014, 28, 241-247. [CrossRef]

68. Xu, L.; Gu, Z.H.; Li, Y.; Zhang, J.L.; Chang, C.K.; Pan, C.M.; Shi, J.Y.; Shen, Y.; Chen, B.; Wang, Y.Y.; et al. Genomic landscape of CD34+ hematopoietic cells in myelodysplastic syndrome and gene mutation profiles as prognostic markers. Proc. Natl. Acad. Sci. USA 2014, 111, 8589-8594. [CrossRef]

69. Forghieri, F.; Paolini, A.; Morselli, M.; Bigliardi, S.; Bonacorsi, G.; Leonardi, G.; Coluccio, V.; Maccaferri, M.; Fantuzzi, V.; Faglioni, L.; et al. NPM1 mutations may reveal acute myeloid leukemia in cases otherwise morphologically diagnosed as myelodysplastic syndromes or myelodysplastic/myeloproliferative neoplasms. Leuk. Lymphoma 2015, 56, 3222-3226. [CrossRef]

70. Cargo, C.A.; Rowbotham, N.; Evans, P.A.; Barrans, S.L.; Bowen, D.T.; Crouch, S.; Jack, A.S. Targeted sequencing identifies patients with preclinical MDS at high risk of disease progression. Blood 2015, 126, 2362-2365. [CrossRef]

71. Peng, J.; Zuo, Z.; Fu, B.; Oki, Y.; Tang, G.; Goswami, M.; Priyanka, P.; Muzzafar, T.; Medeiros, L.J.; Luthra, R.; et al. Chronic myelomonocytic leukemia with nucleophosmin (NPM1) mutation. Eur. J. Haematol. 2016, 96, 65-71. [CrossRef]

72. Bartels, S.; Schipper, E.; Hasemeier, B.; Kreipe, H.; Lehmann, U. Routine clinical mutation profiling using next generation sequencing and a customized gene panel improves diagnostic precision in myeloid neoplasms. Oncotarget 2016, 7, 30084-30093. [CrossRef]

73. Reinig, E.; Yang, F.; Traer, E.; Arora, R.; Brown, S.; Rattray, R.; Braziel, R.; Fan, G.; Press, R.; Dunlap, J. Targeted Next-Generation Sequencing in myelodysplastic syndrome and chronic myelomonocytic leukemia aids diagnosis in challenging cases and identifies frequent spliceosome mutations in transformed acute myeloid leukemia. Am. J. Clin. Pathol. 2016, 145, 497-506. [CrossRef] [PubMed] 
74. Makishima, H.; Yoshizato, T.; Yoshida, K.; Sekeres, M.A.; Radivoyevitch, T.; Suzuki, H.; Przychodzen, B.; Nagata, Y.; Meggendorfer, M.; Sanada, M.; et al. Dynamics of clonal evolution in myelodysplastic syndromes. Nat. Genet. 2017, 49, 204-212. [CrossRef] [PubMed]

75. Vallapureddy, R.; Lasho, T.L.; Hoversten, K.; Finke, C.M.; Ketterling, R.; Hanson, C.; Gangat, N.; Tefferi, A.; Patnaik, M.M. Nucleophosmin 1 (NPM1) mutations in chronic myelomonocytic leukemia and their prognostic relevance. Am. J. Hematol. 2017, 92, e614-e618. [CrossRef] [PubMed]

76. Xu, Y.; Li, Y.; Xu, Q.; Chen, Y.; Lv, N.; Jing, Y.; Dou, L.; Bo, J.; Hou, G.; Guo, J.; et al. Implications of mutational spectrum in myelodysplastic syndromes based on targeted next-generation sequencing. Oncotarget 2017, 8, 82475-82490. [CrossRef]

77. Idossa, D.; Lasho, T.L.; Finke, C.M.; Ketterling, R.P.; Patnaik, M.M.; Pardanani, A.; Gangat, N.; Tefferi, A. Mutations and karyotype predict treatment response in myelodysplastic syndromes. Am. J. Hematol. 2018, 93, 1420-1426. [CrossRef]

78. Hamilton, B.K.; Rybicki, L.; Hirsch, C.; Przychodzen, B.; Nazha, A.; Gerds, A.T.; Hanna, R.; Kalaycio, M.; Sekeres, M.A.; Sobecks, R.; et al. Mutation clonal burden and allogeneic hematopoietic cell transplantation outcomes in acute myeloid leukemia and myelodysplastic syndromes. Bone Marrow Transplant. 2019, 54, 1281-1286. [CrossRef]

79. Montalban-Bravo, G.; Kanagal-Shamanna, R.; Sasaki, K.; Patel, K.; Ganan-Gomez, I.; Jabbour, E.; Kadia, T.; Ravandi, F.; DiNardo, C.; Borthakur, G.; et al. NPM1 mutations define a specific subgroup of MDS and MDS/MPN patients with favorable outcomes with intensive chemotherapy. Blood Adv. 2019, 3, 922-933. [CrossRef]

80. Hwang, S.M.; Kim, S.M.; Nam, Y.; Kim, J.; Kim, S.; Ahn, Y.O.; Park, Y.; Yoon, S.S.; Shin, S.; Kwon, S.; et al. Targeted sequencing aids in identifying clonality in chronic myelomonocytic leukemia. Leuk. Res. 2019, 84, 106190. [CrossRef]

81. Zheng, G.; Chen, P.; Pallavajjalla, A.; Haley, L.; Gondek, L.; Dezern, A.; Ling, H.; De Marchi, F.; Lin, M.T.; Gocke, $\mathrm{C}$. The diagnostic utility of targeted gene panel sequencing in discriminating etiologies of cytopenia. Am. J. Hematol. 2019, 94, 1141-1148. [CrossRef] [PubMed]

82. Vantyghem, S.; Peterlin, P.; Thépot, S.; Ménard, A.; Dubruille, V.; Debord, C.; Guillaume, T.; Garnier, A.; Le Bourgeois, A.; Wuilleme, S.; et al. Diagnosis and prognosis are comforted by integrated assessment of next-generation sequencing in chronic myeloid malignancies. A real-life study. Haematologica 2020. [CrossRef]

83. Wang, K.; Zhou, F.; Cai, X.; Chao, H.; Zhang, R.; Chen, S. Mutational landscape of patients with acute myeloid leukemia or myelodysplastic syndromes in the context of RUNX1 mutation. Hematology 2020, 25, 211-218. [CrossRef] [PubMed]

84. Yu, J.; Li, Y.; Li, T.; Li, Y.; Xing, H.; Sun, H.; Sun, L.; Wan, D.; Liu, Y.; Xie, X.; et al. Gene mutational analysis by NGS and its clinical significance in patients with myelodysplastic syndrome and acute myeloid leukemia. Exp. Hematol. Oncol. 2020, 9, 2. [CrossRef] [PubMed]

85. Wu, L.; Li, X.; Xu, F.; Wu, D.; He, Q.; Song, L.; Xiao, C.; Zhao, Y.; Zhang, Z.; Guo, J.; et al. NPM1 mutation with DNMT3A wild type defines a subgroup of MDS with particularly favorable outcomes after decitabine therapy. Br. J. Haematol. 2020, 198, 982-984. [CrossRef]

86. Yun, S.; Geyer, S.M.; Komrokji, R.S.; Al Ali, N.H.; Song, J.; Hussaini, M.; Sweet, K.L.; Lancet, J.E.; List, A.F.; Padron, E.; et al. Prognostic significance of serial molecular annotation in myelodysplastic syndromes (MDS) and secondary acute myeloid leukemia (sAML). Leukemia 2020. [CrossRef] [PubMed]

87. Patel, S.S.; Ho, C.; Ptashkin, R.N.; Sadigh, S.; Bagg, A.; Geyer, J.T.; Xu, M.L.; Prebet, T.; Mason, E.F.; Seegmiller, A.C.; et al. Clinicopathologic and genetic characterization of nonacute NPM1-mutated myeloid neoplasms. Blood Adv. 2019, 3, 1540-1545. [CrossRef] [PubMed]

88. Patel, S.S.; Kluk, M.J.; Weinberg, O.K. NPM1 biology in myeloid neoplasia. Curr. Hematol. Malig. Rep. 2020, 15, 350-359. [CrossRef] 
89. Greenberg, P.L.; Stone, R.M.; Al-Kali, A.; Barta, S.K.; Bejar, R.; Bennett, J.M.; Carraway, H.; De Castro, C.M.; Deeg, H.J.; DeZern, A.E.; et al. Myelodysplastic Syndromes. NCCN Clinical Practice Guidelines in Oncology. J. Natl. Compr. Cancer Netw. 2017, 15, 60-87, (Updated Version 2. 28 February 2020). [CrossRef]

90. Itzykson, R.; Fenaux, P.; Bowen, D.; Cross, N.C.P.; Cortes, J.; De Witte, T.; Germing, U.; Onida, F.; Padron, E.; Platzbecker, U.; et al. Diagnosis and treatment of chronic myelomonocytic leukemia in adults: Recommendations from the European Hematology Association and the European LeukemiaNet. HemaSphere 2018, 2, e150. [CrossRef]

91. Palomo, L.; Meggendorfer, M.; Hutter, S.; Twardziok, S.; Adema, V.; Fuhrmann, I.; Fuster-Tormo, F.; Xicoy, B.; Zamora, L.; Acha, P.; et al. Molecular landscape and clonal architecture of adult myelodysplastic/myeloproliferative neoplasms. Blood 2020, 136, 1851-1862. [CrossRef] [PubMed]

92. Kennedy, J.A.; Ebert, B.L. Clinical implications of genetic mutations in myelodysplastic syndrome. J. Clin. Oncol. 2017, 35, 968-974. [CrossRef] [PubMed]

93. Hellstrom-Lindberg, E.; Tobiasson, M.; Greenberg, P. Myelodysplastic syndromes: Moving towards personalized management. Haematologica 2020, 105, 1765-1779. [CrossRef]

94. Caponetti, G.C.; Bagg, A. Mutations in myelodysplastic syndromes: Core abnormalities and CHIPing away at the edges. Int. J. Lab. Hematol. 2020. [CrossRef] [PubMed]

95. Malcovati, L.; Hellström-Lindberg, E.; Bowen, D.; Adès, L.; Cermak, J.; Del Cañizo, C.; Della Porta, M.G.; Fenaux, P.; Gattermann, N.; Germing, U.; et al. Diagnosis and treatment of primary myelodysplastic syndromes in adults: Recommendations from European LeukemiaNet. Blood 2013, 122, 2943-2964. [CrossRef]

96. Palomo, L.; Ibáñez, M.; Abáigar, M.; Vázquez, I.; Álvarez, S.; Cabezón, M.; Tazón-Vega, B.; Rapado, I.; Fuster-Tormo, F.; Cervera, J.; et al. Spanish Guidelines for the use of targeted deep sequencing in myelodysplastic syndromes and chronic myelomonocytic leukaemia. Br. J. Haematol. 2020, 188, 605-622. [CrossRef]

97. Hospital, M.A.; Vey, N. Myelodysplastic syndromes: How to recognize risk and avoid acute myeloid leukemia transformation. Curr. Oncol. Rep. 2020, 22, 4. [CrossRef]

98. Menssen, A.J.; Walter, M.J. Genetics of progression from MDS to secondary leukemia. Blood 2020, 136, 50-60. [CrossRef]

99. Bejar, R. What biologic factors predict for transformation to AML? Best Pract. Res. Clin. Haematol. 2018, 31, 341-345. [CrossRef]

100. Fenaux, P.; Platzbecker, U.; Ades, L. How to manage adults with myelodysplastic syndrome. Br. J. Haematol. 2020, 189, 1016-1027. [CrossRef]

101. Patnaik, M.M.; Tefferi, A. Chronic myelomonocytic leukemia: 2018 update on diagnosis, risk stratification and management. Am. J. Hematol. 2018, 93, 824-840. [CrossRef] [PubMed]

102. Pasqualucci, L.; Liso, A.; Martelli, M.P.; Bolli, N.; Pacini, R.; Tabarrini, A.; Carini, M.; Bigerna, B.; Pucciarini, A.; Mannucci, R.; et al. Mutated nucleophosmin detects clonal multilineage involvement in acute myeloid leukemia: Impact on WHO classification. Blood 2006, 108, 4146-4155. [CrossRef] [PubMed]

103. Falini, B.; Macijewski, K.; Weiss, T.; Bacher, U.; Schnittger, S.; Kern, W.; Kohlmann, A.; Klein, H.U.; Vignetti, M.; Piciocchi, A.; et al. Multilineage dysplasia has no impact on biologic, clinicopathologic, and prognostic features of AML with mutated nucleophosmin (NPM1). Blood 2010, 115, 3776-3786. [CrossRef] [PubMed]

104. Díaz-Beyá, M.; Rozman, M.; Pratcorona, M.; Torrebadell, M.; Camós, M.; Aguilar, J.L.; Esteve, J. The prognostic value of multilineage dysplasia in de novo acute myeloid leukemia patients with intermediate-risk cytogenetics is dependent on NPM1 mutational status. Blood 2010, 116, 6147-6148. [CrossRef] [PubMed]

105. Cheng, K.; Sportoletti, P.; Ito, K.; Clohessy, J.G.; Teruya-Feldstein, J.; Kutok, J.L.; Pandolfi, P.P. The cytoplasmic NPM mutant induces myeloproliferation in a transgenic mouse model. Blood 2010, 115, 3341-3345. [CrossRef]

106. Sportoletti, P.; Varasano, E.; Rossi, R.; Mupo, A.; Tiacci, E.; Vassiliou, G.; Martelli, M.P.; Falini, B. Mouse models of NPM1-mutated acute myeloid leukemia: Biological and clinical implications. Leukemia 2015, 29, 269-278. [CrossRef]

107. Guryanova, O.A.; Shank, K.; Spitzer, B.; Luciani, L.; Koche, R.P.; Garrett-Bakelman, F.E.; Ganzel, C.; Durham, B.H.; Mohanty, A.; Hoermann, G.; et al. DNMT3A mutations promote anthracycline resistance in acute myeloid leukemia via impaired nucleosome remodeling. Nat. Med. 2016, 22, 1488-1495. [CrossRef]

108. Mupo, A.; Celani, L.; Dovey, O.; Cooper, J.L.; Grove, C.; Rad, R.; Sportoletti, P.; Falini, B.; Bradley, A.; Vassiliou, G.S. A powerful molecular synergy between mutant nucleophosmin anf Flt3-ITD drives acute myeloid leukemia in mice. Leukemia 2013, 27, 1917-1920. [CrossRef] 
109. Mallardo, M.; Caronno, A.; Pruneri, G.; Raviele, P.R.; Viale, A.; Pelicci, P.G.; Colombo, E. NPMc+ and FLT3-ITD mutations cooperate in inducing acute leukaemia in a novel mouse model. Leukemia 2013, 27, 2248-2251. [CrossRef]

110. Dovey, O.M.; Cooper, J.L.; Mupo, A.; Grove, C.S.; Lynn, C.; Conte, N.; Andrews, R.M.; Pacharne, S.; Tzelepis, K.; Vijayabaskar, M.S.; et al. Molecular synergy underlies the co-occurrence patterns and phenotype of NPM1-mutant acute myeloid leukemia. Blood 2017, 130, 1911-1922. [CrossRef]

111. Falini, B.; Sportoletti, P. A scale of "bad" co-mutations in NPM1-driven AML. Blood 2017, 130, 1877-1879. [CrossRef] [PubMed]

112. Loberg, M.A.; Bell, R.K.; Goodwin, L.O.; Eudy, E.; Miles, L.A.; SanMiguel, J.M.; Young, K.; Bergstrom, D.E.; Levine, R.L.; Schneider, R.K.; et al. Sequentially inducible mouse models reveal that Npm1 mutation causes malignant transformation of Dnmt3a-mutant clonal hematopoiesis. Leukemia 2019, 33, 1635-1649. [CrossRef] [PubMed]

113. Uckelmann, H.J.; Kim, S.M.; Wong, E.M.; Hatton, C.; Giovinazzo, H.; Gadrey, J.Y.; Krivtsov, A.V.; Rucker, F.G.; Dohner, K.; McGeehan, G.M.; et al. Therapeutic targeting of preleukemia cells in a mouse model of NPM1 mutant acute myeloid leukemia. Science 2020, 367, 586-590. [CrossRef] [PubMed]

114. Forghieri, F.; Nasillo, V.; Colasante, P.; Potenza, L.; Luppi, M. Are We Ready to Fight Against NPM1-Mutated Preleukemic Clones in Humans? Available online: https://science.sciencemag.org/content/367/6477/586/tabe-letters (accessed on 27 February 2020).

115. Jaiswal, S.; Ebert, B.L. Clonal hematopoiesis in human aging and disease. Science 2019, 366, eaan4673. [CrossRef]

116. Shlush, L.I.; Zandi, S.; Mitchell, A.; Chen, W.C.; Brandwein, J.M.; Gupta, V.; Kennedy, J.A.; Schimmer, A.D.; Schuh, A.C.; Yee, K.W.; et al. Identification of pre-leukaemic haematopoietic stem cells in acute leukaemia. Nature 2014, 506, 328-333. [CrossRef]

117. Patel, J.L.; Schumacher, J.A.; Frizzell, K.; Sorrells, S.; Shen, W.; Clayton, A.; Jattani, R.; Kelley, T.W. Coexisting and cooperating mutations in NPM1-mutated acute myeloid leukemia. Leuk. Res. 2017, 56, 7-12. [CrossRef]

118. Potter, N.; Miraki-Moud, F.; Ermini, L.; Titley, I.; Vijayaraghavan, G.; Papaemmanuil, E.; Campbell, P.; Gribben, J.; Taussig, D.; Greaves, M. Single cell analysis of clonal architecture in acute myeloid leukaemia. Leukemia 2019, 33, 1113-1123. [CrossRef]

119. Herudkova, Z.; Culen, M.; Folta, A.; Jeziskova, I.; Cerna, J.; Loja, T.; Tom, N.; Smejkal, J.; Semerad, L.; Dvorakova, D.; et al. Clonal hierarchy of main molecular lesions in acute myeloid leukaemia. Br. J. Haematol. 2019. [CrossRef]

120. van Galen, P.; Hovestadt, V.; Wadsworth Ii, M.H.; Hughes, T.K.; Griffin, G.K.; Battaglia, S.; Verga, J.A.; Stephansky, J.; Pastika, T.J.; Lombardi Story, J.; et al. Single-cell RNA-Seq reveals AML hierarchies relevant to disease progression and immunity. Cell 2019, 176, 1265-1281. [CrossRef]

121. Krönke, J.; Bullinger, L.; Teleanu, V.; Tschürtz, F.; Gaidzik, V.I.; Kühn, M.W.; Rücker, F.G.; Holzmann, K.; Paschka, P.; Kapp-Schwörer, S.; et al. Clonal evolution in relapsed NPM1-mutated acute myeloid leukemia. Blood 2013, 122, 100-108. [CrossRef]

122. Herold, S.; Sockel, K.; Sayehli, C.; Herbst, R.; Dührsen, U.; Oelschlägel, U.; Böttner, A.; Hindahl, H.; Kullmer, J.; Helas, S.; et al. Evolution of NPM1-negative therapy-related myelodysplastic syndromes following curative treatment of NPM1-mutant AML. Leukemia 2017, 31, 2247-2251. [CrossRef] [PubMed]

123. Cocciardi, S.; Dolnik, A.; Kapp-Schwoerer, S.; Rücker, F.G.; Lux, S.; Blätte, T.J.; Skambraks, S.; Krönke, J.; Heidel, F.H.; Schnöder, T.M.; et al. Clonal evolution patterns in acute myeloid leukemia with NPM1 mutation. Nat. Commun. 2019, 10, 2031. [CrossRef] [PubMed]

124. Klossowski, S.; Miao, H.; Kempinska, K.; Wu, T.; Purohit, T.; Kim, E.; Linhares, B.M.; Chen, D.; Jih, G.; Perkey, E.; et al. Menin inhibitor MI-3454 induces remission in MLL1-rearranged and NPM1-mutated models of leukemia. J. Clin. Investig. 2020, 130, 981-997. [CrossRef] [PubMed]

125. Falini, B.; Brunetti, L.; Martelli, M.P. Dactinomycin in NPM1-mutated acute myeloid leukemia. N. Engl. J. Med. 2015, 373, 1180-1182. [CrossRef] [PubMed] 
126. Beziat, G.; Tavitian, S.; Bertoli, S.; Huguet, F.; Largeaud, L.; Luquet, I.; Vergez, F.; Rieu, J.B.; Bories, P.; Delabesse, E.; et al. Dactinomycin in acute myeloid leukemia with NPM1 mutations. Eur. J. Haematol. 2020, 105, 302-307. [CrossRef]

127. Lachowiez, C.A.; Loghavi, S.; Kadia, T.M.; Daver, N.; Borthakur, G.; Pemmaraju, N.; Naqvi, K.; Alvarado, Y.; Yilmaz, M.; Short, N.; et al. Outcomes of older patients with NPM1-mutated AML: Current treatments and the promise of venetoclax-based regimens. Blood Adv. 2020, 4, 1311-1320. [CrossRef]

128. Kim, T.; Tyndel, M.S.; Kim, H.J.; Ahn, J.S.; Choi, S.H.; Park, H.J.; Kim, Y.K.; Yang, D.H.; Lee, J.J.; Jung, S.H.; et al. The clonal origins of leukemic progression of myelodysplasia. Leukemia 2017, 31, 1928-1935. [CrossRef]

129. Falini, B.; Brunetti, L.; Martelli, M.P. How I diagnose and treat NPM1-mutated AML. Blood 2020. [CrossRef]

Publisher's Note: MDPI stays neutral with regard to jurisdictional claims in published maps and institutional affiliations.

(C) 2020 by the authors. Licensee MDPI, Basel, Switzerland. This article is an open access article distributed under the terms and conditions of the Creative Commons Attribution (CC BY) license (http://creativecommons.org/licenses/by/4.0/). 